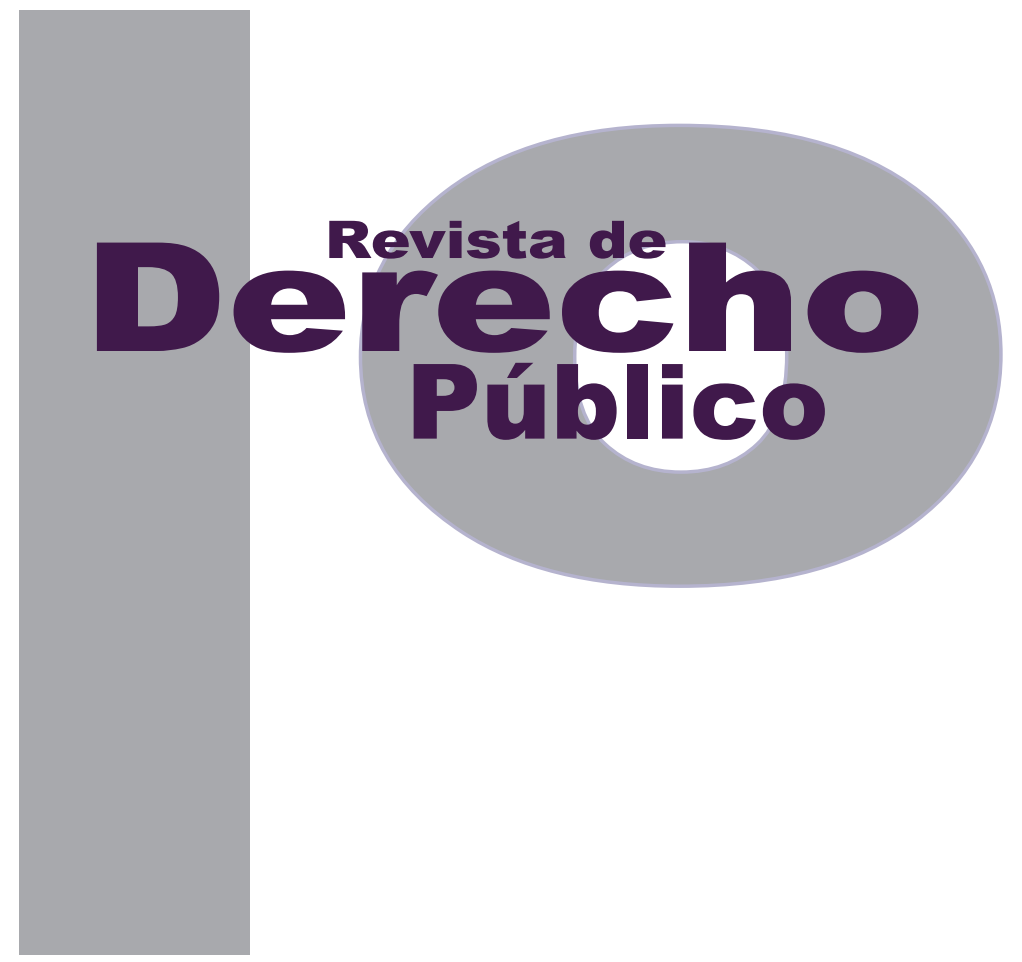

\title{
DE LAS VÍCTIMAS INVISIBLES A LAS VÍCTIMAS DIGNIFICADAS: LOS RETOS DEL ENFOQUE DIFERENCIAL PARA LA POBLACIÓN LGBTI EN LA LEY DE VÍCTIMAS
}

\author{
Mauricio Albarracín Caballero \\ JuAn CARLOS Rincón
}

Universidad de los Andes

Facultad de Derecho

Revista de Derecho Público N. ${ }^{\circ} 31$

Julio - Diciembre de 2013. ISSN 1909-7778 


\title{
De las víctimas invisibles a las víctimas dignificadas: los retos del enfoque diferencial para la población LGBTI en la Ley de Víctimas*
}

\author{
Mauricio Albarracín Caballero** \\ Juan Carlos Rincón***
}

RESUMEN

Este artículo analiza la información sobre la violencia contra la población de lesbianas, gays, bisexuales, transgeneristas e intersexuales (LGBTI) en el marco del conflicto armado, así como la emergencia del enfoque diferencial para esta población en la Ley de Víctimas. En cada uno de estos procesos se evidenciarán los retos que plantean la documentación y la intervención estatal en el fenómeno. Se argumentará que estos dos procesos políticos, el de generación de información y el de la creación legal del enfoque diferencial, intentan fundar un orden social distinto al producido por los actores armados
This paper analyzes the information regarding violence against the lesbian, gay, bisexual, transgender and intersexual (LGBTI) population under Colombia's armed conflict, as well as the emergence of the differential approach for this population in the Law of Victims (Ley de Víctimas). For each of these processes the paper will discuss their creation as well as the challenges posed by their documentation and by state intervention in the phenomenon's. We argue that these two political processes, the generation of information and the legal creation of a differential approach, try to establish a different social order to that

Cómo citar este artículo: Albarracín, M. y Rincón, J. C. (Diciembre, 2013). De las víctimas invisibles a las víctimas dignificadas: los retos del enfoque diferencial para la población LGBTı en la Ley de Víctimas. Revista de Derecho Público, 31.

* Abogado (Universidad Industrial de Santander). Maestría en Derecho (Universidad de los Andes) y maestría en International Legal Studies (American University). Abogado y consultor en derechos de la población LGBTI. Correo: malbarracin@gmail.com

A... Abogado (Universidad de los Andes) con estudios en periodismo. Correo: jkrincon@gmail.com 
contra la población LGBTI. No obstante lo anterior, estos procesos políticos interactúan y presentan retos importantes para la vigencia plena de los derechos de este grupo poblacional. En particular se evidencian desafíos relacionados con la identidad sexual de las víctimas, el tipo de violencia que ejercen contra ellas, los contextos socioculturales en los cuales se produce la violencia y la acción institucional coordinada.

PALABRAs Clave: derechos LgBti, lesbianas, gays, bisexuales, transgeneristas, intersexuales, conflicto armado, víctimas, ley de víctimas, enfoque diferencial. produced by armed parties against the lgbti population. Nonetheless, these political processes interact and pose substantial challenges to the full observance of the rights of this population. In particular, specific challenges are identified regarding the sexual identity of the victims, the type of violence exercised against them, the sociocultural contexts in which violence is produced and the coordinated institutional action.

KEY WORDS: LGBTI rights, lesbians, gays, bisexuals, transgenders, intersexuals, armed conflict, victims, law of victims, differential approach. 


\section{SUMARIO}

Introducción - I. EMERGENCIA DEL DISCURSO ACERCA DE LA VIOLENCIA CONTRA LA POBLACIÓN LGBTI - A. Obstáculos para la investigación de la violencia en el marco del conflicto armado contra la población LGBT - 1. La situación de la víctima y su orientación sexual o identidad de género - 2. El tipo de violencia - 3. El contexto sociocultural - B. Síntesis de la información sobre violencia en el marco del conflicto armado contra la población LGBTI - 1. Cronología de la violencia en el contexto del conflicto armado según el trabajo de Colombia Diversa - II. EMERGENCIA DEL ENFOQUE DIFERENCIAL SOBRE ORIENTACIÓN SEXUAL EN LA LEY DE VÍCTIMAS - III. RETOS PARA EL ENFOQUE DIFERENCIAL Y LA INVESTIGACIÓN SOBRE LA VIOLENCIA CONTRA LA POBLACIÓN LGBTI - IV. CONCLUSIÓN - Bibliografía. 


\section{Introducción}

En el informe "Basta Ya", del Centro Nacional de Memoria Histórica, se hizo un breve análisis sobre la violencia contra lesbianas, gays, bisexuales, transgeneristas e intersexuales (en adelante LGBTI $^{4}$ ) el cual se tituló Degradados, ridiculizados y silenciados. El Grupo de Memoria Histórica no realizó ningún estudio sobre esta población, pero mencionó brevemente el caso de los vejámenes públicos contra la población LGBTI en San Onofre (Sucre), por parte del comandante paramilitar alias "Cadena". El balance general del Grupo de Memoria Histórica parece resumirse en esta afirmación: "en la mayoría de las regiones [el Grupo] se encontró con un profundo silencio sobre lo acontecido con esta población durante el conflicto armado". El mismo informe confirma que esta afirmación evidencia la invisibilidad de esta violencia, la cual ha sido denunciada por las organizaciones LGBTI del país, en particular Colombia Diversa. El caso de San Onofre y otro testimonio citado le sirven al Grupo de Memoria Histórica para formular una

$4 \quad$ Adoptamos en este punto las definiciones de las Directrices sobre protección internacional No. 9 de la Oficina del Alto Comisionado de las Naciones Unidas para los Refugiados (acnur). Ver parte III: Terminología, en http://www.refworld.org/cgi-bin/texis/vtx/rwmain/opendocpdf. pdf?reldoc=y\&docid=518113d54. Al respecto, recogemos la advertencia con que termina dicha sección: "No todos los solicitantes se autoidentifican con la terminología lgbti y los conceptos que se presentaron anteriormente o puede que no conozcan estos términos. Puede que algunos sólo puedan referirse a los términos (despectivos) utilizados por el perseguidor. Las personas encargadas de la toma de decisiones, por tanto, deben tener cuidado de no aplicar estos términos inflexiblemente ya que esto podría resultar en evaluaciones de credibilidad adversas o la falta de reconocimiento de una solicitud válida. Por ejemplo, los bisexuales a menudo son clasificados en la adjudicación de solicitudes de la condición de refugiado como gays, lesbianas o heterosexuales, las personas intersexuales puede que no se identifiquen en absoluto como lgbti (puede ser que no ven su condición como parte de su identidad, por ejemplo) y los hombres que tienen relaciones sexuales con otros hombres no siempre se identifican como gays". Párrafo 11, p. 9. hipótesis: Ios actores armados realizaban actos de violencia contra la población LGBTI, en algunas ocasiones con gran publicidad, para establecer órdenes sociales autoritarios. El informe también señala que “[e]n esa cultura machista y patriarcal se inscriben formas conservadoras de concebir la sexualidad que llevan a los actores armados a desterrar, perseguir y humillar a poblaciones con opciones sexuales que estos consideran transgresoras". ${ }^{5}$

La violencia institucional o social participa en la constitución de la orientación sexual e identidad de género. Esta violencia tuvo dimensiones estructurales con la penalización de la sodomía bajo el régimen inquisitorial y posteriormente de la homosexualidad en tiempos republicanos. Incluso Marcel Proust llegó a decir que “los homosexuales son una raza maldita, perseguida como Israel. Y finalmente, como Israel, bajo el oprobio de un odio inmerecido por parte de las masas, adquirieron características de masa, la fisonomía de una nación (...) son en cada país una colonia extranjera". ${ }^{6}$ En el mismo sentido, el sociólogo Didier Eribon ha hecho énfasis en el papel de la injuria como acto constitutivo de la identidad homosexual:

En el principio hay la injuria. La que cualquier gay puede oír en un momento u otro de su vida, y que es el signo de su vulnerabilidad psicológica y social. La injuria no es solamente una palabra que describe. No se conforma con anunciarme lo que soy. Si alguien me tacha de

5 GRUPO DE MEMORIA HISTÓRICA. ¡Basta ya! Colombia: Memorias de guerra y dignidad. Bogotá: Imprenta Nacional, 2013, p. 322.

$6 \quad$ PROUST, Marcel. En busca del tiempo perdido. 1913. 
"sucio marica" o incluso lisa y llanamente de marica, no trata de comunicarme una información sobre mí mismo. El que lanza el ultraje me hace saber que tiene el poder sobre mí, que estoy a su merced... La injuria es un acto de lenguaje -o una serie repetida de actos- por el cual se asigna a su destinatario un lugar en el mundo. La injuria produce efectos profundos en la conciencia de un individuo porque le dice: "te asimilo a", "te reduzco a". ${ }^{7}$

Siguiendo con esta línea de razonamiento, Pierre Bourdieu evidenció cómo la forma de dominación o violencia simbólica ejercida contra las personas LGBTI es realizada mediante actos colectivos de categorización que diferencian los sujetos y los marcan negativamente. No en vano, nos recuerda Bourdieu, categoría proviene del griego Kategoría que significa acusación pública. Los actos de categorización están formados por actos de rechazo puro, ironía, injuria, de escarnio público; además de actos del lenguaje hay actos de eliminación física como los crímenes de odio, robos, homicidios, violaciones de derechos humanos motivados por la orientación sexual, y los actos de exclusión de los espacios sociales. ${ }^{8}$

Estos actos de categorización instauran la opresión, entendida como invisibilización, que se manifiesta en la existencia legítima y pública de la homosexualidad. Además, la invisibilización tiene su correlativa exigencia en los sujetos dominados: la discreción, el disimulo y, en general,

7 ERIBON, Didier. Reflexiones sobre la cuestión gay. Barcelona: Anagrama, 2001.

8 BOURDIEU, Pierre. La dominación masculina. Barcelona: Anagrama, 2000 , p. 143 a 149. desaparecer de la vida social. La invisibilidad es la forma de violencia simbólica contra la población LGBTI, entendida como una "violencia amortiguada, insensible, e invisible para sus propias víctimas, que se ejerce esencialmente a través de caminos puramente simbólicos de la comunicación y del conocimiento o más exactamente del desconocimiento, del reconocimiento o, en último término, del sentimiento". ${ }^{9}$ Esta invisibilidad está acompañada por la vergüenza, que es el mecanismo por el cual se perpetúa la discreción. Como afirma Camila Esguerra: "La vergüenza, causarla o sentirla, es (...) una forma de eliminación simbólica". ${ }^{10}$

La violencia tiene una dimensión expresiva que constituye las identidades de las personas LGBTI y produce y reproduce el silencio, la invisibilidad y la vergüenza. Esta represión también genera agencias y movimientos que reaccionan a la injusticia, a través de la visibilidad y el orgullo. De esta manera, una identidad menospreciada se reconstituye en una identidad dignificada.

En esta tensión entre identidades menospreciadas y dignificadas se ubica el debate sobre la violencia contra las personas LGBTI en el conflicto armado. La invisibilidad y el silencio en torno a la violencia contra las personas LGBTI en el conflicto armado es la continuación de este orden social autoritario: una violencia nefanda, como lo fue en la edad media el pecado que no osaba decir su nombre. Esta violencia tiene un rol constitu-

\footnotetext{
$9 \quad$ Ibíd., p. 36.

10 ESGUERRA, Camila. A propósito de la vergüenza en prácticas artísticas enfoques contemporáneos. Universidad Nacional -IDCT, 2003.
} 
tivo en las identidades de las personas LGBTI de las regiones donde ocurre, así como de las personas heterosexuales. La violencia es producida para establecer un orden social, por ello tiene el efecto de eliminar al marginal y disciplinar a la sociedad. Por tanto, la violencia de actores armados contra la población LGBTI es un acto performativo que trata de refundar un orden social, moral y político, es decir, un nuevo nomos.

A pesar del ocultamiento de la violencia, las organizaciones LGBTI han iniciado un esfuerzo colectivo en la última década por hacer visible esta violencia a través de la denuncia y la documentación. El movimiento LGBTI replicó las estrategias de los grupos de derechos humanos con el fin de dignificar a sus muertos y producir un cambio institucional que impactara en la prevención, investigación y sanción de estos hechos. De esta manera, aparece la emergencia del discurso acerca de la violencia contra la población LGBTI. El movimiento LGBTI también intenta refundar un orden social, moral y político. Tal y como lo ha mostrado Julieta Lemaitre, se trata de dos proyectos políticos en disputa: el nomos de la violencia y el nomos de los derechos. ${ }^{11}$

En este contexto de confrontación de proyectos políticos aparece la Ley 1448 de $2011,{ }^{12}$ conocida popularmente como Ley de Víctimas. En esta Ley se introducen tres normas que hacen

11 LEMAITRE RIPOLL, Julieta. El amor en tiempos de cólera: derechos LGBT en Colombia. En: SUR. Revista Internacional de Derechos Humanos, vol. 6, no. 11, Dic. 2009

12 "Por la cual se dictan medidas de atención, asistencia y reparación integral a las víctimas del conflicto armado interno y se dictan otras disposiciones". Ley 1448 de 2011. visibles a las víctimas LGBTI del conflicto armado: (i) el reconocimiento de las parejas del mismo sexo como víctimas (artículo 3); (ii) la mención expresa de la orientación sexual dentro del principio de igualdad (artículo 6); y (iii) el establecimiento de la orientación sexual como parte del enfoque diferencial (artículo 13). En esta ley se produce la emergencia del enfoque diferencial sobre orientación sexual.

Este artículo analiza los procesos políticos del discurso acerca de la violencia contra la población LGBTI y la emergencia del enfoque diferencial para esta población en la Ley de Víctimas. En cada uno de estos procesos se evidenciará su creación y los retos que plantea. Se argumentará que estos dos procesos políticos, el de generación de información y el de la creación legal del enfoque diferencial, intentan fundar un orden social distinto al producido por los actores armados contra la población LGBTI. No obstante lo anterior, estos procesos políticos interactúan y presentan retos importantes para la vigencia plena de los derechos. La principal paradoja es que la violencia contra la población LGBTı ha aumentado en los últimos años coincidiendo con la vigencia de la Ley 1448 de 2011, como se expondrá más adelante.

\section{EMERGENCIA DEL DISCURSO ACERCA DE LA VIOLENCIA CONTRA LA POBLACIÓN LGBTI}

En esta sección se analiza la emergencia del proceso político de documentación de la violencia contra la población LGBTI, realizado por las 
organizaciones de este movimiento social en los últimos diez años. Organizaciones como Colombia Diversa, Caribe Afirmativo, la Fundación Santamaría de Cali, entre otras, han iniciado un proceso de denuncia y documentación que tiene un objetivo común: mostrar la violencia que ha sido silenciada por las condiciones socioculturales en las cuales se produjo. A continuación se mostrarán los obstáculos que tiene la investigación de la violencia contra la población LGBTI en el marco del conflicto armado, y seguidamente el proceso de reporte que viene haciendo Colombia Diversa, organización pionera en este campo, de forma sistemática, desde el año 2004.

\section{A. Obstáculos para la investigación de la violencia en el marco del conflicto arma- do contra la población LGBT ${ }^{13}$}

En el contexto internacional se han documentado situaciones de conflicto armado o regímenes autoritarios en los cuales se ha ejercido violencia contra la población LGBTI. Son muy conocidos los hechos de persecución y exterminio en la Alemania nazi, ${ }^{14}$ la persecución del régimen franquista en España, la del régimen castrista en

13 La sección correspondiente a los obstáculos para la investigación, y una parte del balance de la información, fue publicada previamente en ALBARRACíN, Mauricio. Desplazamiento forzado contra las personas LGBT: reflexiones para la investigación e implementación de políticas públicas. En: Colombia Diversa. Todos los deberes, pocos los derechos: situación de las personas lesbianas, gay, bisexuales y transgeneristas 2008-2009". Bogotá, 2011, p. 131-145. Se reproduce una versión ajustada de ese texto con autorización de la organización Colombia Diversa.

14 Véase GILES, Geoffrey J. Legislating Homophobia in the Third Reich: The Radicalization of Prosecution Against Homosexuality by the Legal Profession. En: German History, 2005, vol. 3, n. 23, p. 339-354. Bibliografía disponible sobre estos hechos en: http://www.ushmm.org/ research/library/bibliography/index.php?content=gays.
Cuba $^{15}$ y la del conflicto armado en Perú. En España y Perú, las personas LGBTI víctimas del conflicto armado fueron reconocidas, en el primer caso mediante la Ley de Memoria Histórica, ${ }^{16}$ y en el segundo a través de la Comisión de la Ver$\operatorname{dad}^{17}$. Un área que ha tenido mayor desarrollo es la investigación y análisis de situaciones de personas LGBTI que solicitan refugio en diversos países, especialmente en la determinación de la pertenencia al grupo vulnerable y la conexidad entre la amenaza y el refugio. ${ }^{18}$ La Agencia de la

15 EPPS, Bradley S. Proper Conduct: Reinaldo Arenas, Fidel Castro, and the Politics of Homosexuality. En: Journal Of the History of Sexuality, $1995, n^{\circ} .66$, p. 232-33.

16 Artículo 2, numeral 2 de la Ley de Memoria Histórica de España. Ley $52 / 2007$ del 26 de diciembre, por la que se reconocen y amplían derechos y se establecen medidas en favor de quienes padecieron persecución o violencia durante la Guerra Civil y la dictadura.

Artículo 2. Reconocimiento general.

“1. Como expresión del derecho de todos los ciudadanos a la reparación moral y a la recuperación de su memoria personal y familiar, se reconoce y declara el carácter radicalmente injusto de todas las condenas, sanciones y cualesquiera formas de violencia personal producidas por razones políticas, ideológicas o de creencia religiosa, durante la Guerra Civil, así como las sufridas por las mismas causas durante la Dictadura".

"2. Las razones a las que se refiere el apartado anterior incluyen [...] el ejercicio de conductas vinculadas con opciones culturales, lingüísticas o de orientación sexual [...]".

Véase Informe final de la Comisión de la Verdad y Reconciliación. Tomo II. Primera parte: El proceso, los hechos, las víctimas. Sección segunda: Los actores del conflicto, Capítulo 1: Los actores armados, 1.4. El Movimiento Revolucionario Túpac Amaru, 1.4.3. Actos de terror contra minorías sexuales. Perú, 2003.

18

BERG, Laurie y MILLBANK, Jenni. Constructing the Personal Narratives of Lesbian, Gay and Bisexual Asylum Claimants. En: Journal of Refugee Studies, 2009, vol. 22, n. 2, p. 195-223. DAUVERGNE, Catherine y MILLBANK, Jenni. Burdened by Proof: How the Australian Refugee Review Tribunal Has Failed Lesbian and Gay Asylum Seekers. En: Federal Law Review, 2003, nº. 31, p. 299-342. HANNA, Fadi. Punishing Masculinity in Gay Asylum Claims. En: The Yale Law Journal, 2005, nº 114 , p. 913. Informe de la Alta Comisionada de las Naciones Unidas para los Derechos Humanos sobre la situación de los derechos humanos en Colombia, 2009. 4 de marzo del 2010. FAIRBURN, Bill. Gay Rights Are Human Rights: Gay Asylum Seekers in Canada. En: Brad, E.; Keja, V. and Bill Johnson, G. (editors). Passing Lines: Sexuality and Immigration. Cambridge, MA: David Rockefeller Center for Latin American Studies, Harvard University, 2005, pp. 237- 
ONU para los Refugiados (ACNUR) ha desarrollado una serie de estudios que identifican los obstáculos que afrontan los asilados miembros de la población LGBTI, en los que proponen herramientas metodológicas para solucionarlos. Estos casos son únicos, razón por la cual no existe un cuerpo teórico ni metodológico muy desarrollado que permita acercarse a la documentación y al análisis de la violencia contra la población LGBTI en el contexto de conflictos armados. ${ }^{19}$

Recientemente ha surgido una preocupación por la violencia sexual contra los hombres en contextos de conflictos armados. Denuncia Dustin Lewis, por ejemplo, que "la violencia sexual contra los hombres como constitutiva del genocidio, crímenes contra la humanidad, y crímenes de guerra, usualmente pasa desapercibida, no es procesada y, en últimas, no recibe castigo". ${ }^{20}$ Sandesh Sivakumaran argu-

252. KENDALL, Christopher N. Lesbian and Gay Refugees in Australia: Now that "Acting Discreetly" Is No longer an Option, Will Equality Be Forthcoming? En: International Journal of Refugee Law, 2005, vol. 15, $n^{\circ} .4$, p. 715. MCGHEE, Derek. Persecution and Social Group Status: Homosexual Refugees in the 1990s. 14 J. Refugee Studies 20, 2001. MILLBANK, Jenni. Constructing the Personal Narratives of Lesbian, Gay, and Bisexual Asylum Claimants. En: Journal of Refugee Studies, 2009. REHAAG, Sean. Patrolling the Borders of Sexual Orientation: Bisexual Refugee Claims in Canada. En: McGill Law Journal 53, 2008, p. 70. SWINK, Arwen. Queer Refuge: A Review of the Role of Country Condition Analysis in Asylum Adjudications for Members of Sexual Minorities. En: 29 Hastings Int'l \& Comp. L. Rev. 2006, p. 251.

19 Las investigaciones sobre violencia contra la población Igbti se han concentrado más en sociedades en situaciones de paz, por ejemplo sobre crímenes de odio o violencia homofóbica. Esta literatura puede dar luces para analizar la violencia en el conflicto armado, pero es insuficiente para analizar los contextos de violencia generalizada. Existe una serie de publicaciones que trata el tema de cómo atender a los refugiados y desplazados miembros de la población Igbti. Entre ellos, véase U. S. CITIZENSHIP AND INMIGRATION SERVICES. Guidance for adjudicating Lesbian, Gay, Bisexual, Transgender, and Intersex (Igbti) refugee and asylum claims, 2011; UNHCR-UNHCR. Guidance note on refugee claims relating to sexual orientation and gender identity, 2008.

20 La traducción es nuestra. El texto en inglés dice así: "Sexual violence against men as a constitutent element of genocide, crimes against menta que la violación de un hombre a otro, por ejemplo, es un tema que no suele tratarse a nivel internacional, principalmente porque las sociedades, dada su homofobia, suelen asociar la violación con una conducta homosexual, sin tener en cuenta la coerción que se presenta. ${ }^{21}$ Reconociendo los avances que se han obtenido a partir del enfoque diferencial de género en los contextos de violencia, en la doctrina se ha propuesto, como lo expresa Charli Carpenter, que "las referencias a la violencia de género contra las mujeres y niñas en el conflicto son inseparables de un estudio de las formas de violencia a las que los hombres civiles son especialmente vulnerables".22 Sobre el tema, analizando específicamente la guerra de la antigua Yugoslavia, Dubravka Sarkov advierte que "tomar a los hombres siempre y únicamente como violadores, y nunca como víctimas de violación y otras formas de violencia sexual", ${ }^{23}$ es un error que

humanity, and war crimes often goes under noticed, under prosecuted and, ultimately, under punished." LEWIS, Dustin. Unrecognized Victims: Sexual Violence Against Men in Conflict Settings Under International Law. En: Wisconsin International Law Journal, 2009.

21 SIVAKUMARAN, Sandesh. Male/Male Rape and the "Taint" of Homosexuality. Human Rights Quarterly, 2005.

22 La traducción es nuestra. El texto en inglés dice: "addressing genderbased violence against women and girls in conflict situations is inseparable from addressing the forms of violence to which civilian men are specifically vulnerable." CARPENTER, Charli. Recognizing GenderBased Violence Against Civilian Men and Boys in Conflict Situations. En: Security Dialogue, March 2006, vol. 37, p. 183-103. Disponible en: http://sdi.sagepub.com/content/37/1/83.short. SOUTH AFRICAN MEDICAL RESEARCH COUNCIL. Briefing Paper. Care and Support of Male Survivors of Conflict-Related Sexual Violence. Sexual Violence Research Initiative. 2011. Disponible en: http://www.svri.org/CareSupportofMaleSurviv.pdf, denuncia lo mismo.

23 La traducción es nuestra. ZARKOV, Dubrabvka. The Body of The Other Man. Sexual violence and the Construction of Masculinity, Sexuality and Ethnicity in Croatian Media. En: MOSER, C. y CLARK, F. Victims, Perpretators, or actors? Gender, Armed Conflict and Political Violence. Capítulo 5. Zed Books. Disponible en: http://www.umass. edu/wost/syllabi/spring06/zarkov.pdf 
invisibiliza a una porción de las víctimas y se encarga de perpetuar nociones heterosexuales erradas sobre el conflicto. En su estudio del mismo caso, Pauline Oosterhoff, Prisca Zwanikken y Evert Ketting denuncian la utilización de la tortura sexual contra los hombres como instrumento de guerra, ${ }^{24}$ mientras que Hilmi Zawati, quien se ocupa del caso Iraquí, concluye que "la violación masculina, en tiempos de guerra, es predominantemente una demostración de poder y una agresión, no un intento para satisfacer el deseo sexual. El efecto que un ataque tan horrible busca es dañar la psiquis de la víctima, robarle su orgullo e intimidarla". ${ }^{25}$ Por su parte, Kristine Eck y Olga Vera desmitifican la noción de que las mujeres son las principales víctimas en los conflictos armados, y proponen que son los hombres quienes se encuentran más vulnerables en estos contextos. ${ }^{26}$ En un informe

24 Cuentan los autores que pocos hombres admiten haber sido torturados sexualmente o buscan ayuda, y es posible que los profesionales no reconozcan los casos. Se han procesado pocos perpetradores, por falta de voluntad política. El silencio que cubre la tortura sexual de hombres en la posguerra en Croacia es un contraste extraño ante el carácter público de los crímenes mismos." OOSTERHOFF, Pauline, ZWANIKKEN, Prisca y KETTING, Evert. Sexual Torture of Men in Croatia and Other Conflict Situations: An Open Secret. En: Reproductive Health Matters, 2004, vol. 12, issue 23, p. 68-77. Disponible en: http://www.rhm-elsevier.com/article/S0968-8080(04)23115-9/abstract

25 La traducción es nuestra. Texto en inglés: "Male rape, in time of war, is predominantly an assertion of power and aggression rather than an attempt on the part of the perpetrator to satisfy sexual desire. The effect of such a horrible attack is to damage the victim's psyche, rob him of his pride, and intimidate him." ZAWATI, Hilmi. Impunity or immunity: wartime male rape and sexual torture as a crime against humanity. En: Journal on Rehabilitation of Torture Victims and Prevention of Torture, p. 27-47. Disponible en: http://www.irct.org/Files/Filer/TortureJournal/17_1_2007/impunity_or_immunity.pdf También, en un sentido más amplio, Sandesh Sivakumaran.

Dicen las autoras que "sensibilizar sobre el victimismo masculino puede ser una clave estratégica para obtener un cambio en los roles de género. Mientras que los hombres no sean vistos - ni se vean a sí mismos- como víctimas de un rol de género desigual, pocos de ellos participarán en la reconstrucción activa de la hegemonía masculina". La traducción es nuestra. ECK, Kristine y HÄNNI, Olga. Male Victimhood sobre las medidas que adopta la Organización de Naciones Unidas para atender los hombres y niños víctimas de violencia sexual, Sandesh Sivakumaran dice que hay problemas que deben solucionarse con respecto a la visibilización y sensibilización del problema, al estudio de la relación entre la violencia contra hombres y niños y la violencia contra mujeres y niñas, al entendimiento de la violencia contra los niños, y a la situación del hombre como víctima y la mujer como perpetradora. ${ }^{27} \mathrm{El}$ silencio que ha rodeado históricamente el tema parece comenzar a ser atendido, pero aún faltan muchos más estudios sobre la violencia sexual contra los hombres en los conflictos armados.

En Colombia se han iniciado estudios sobre el conflicto armado y su impacto sobre la población LGBTI. Nancy Prada coordinó un esfuerzo de la Escuela de Estudios de Género, de la Facultad de Ciencias Humanas de la Universidad Nacional de Colombia (sede Bogotá), por realizar memoria histórica incluyente mediante el relato de historias de mujeres trans que se desplazaron forzosamente hacia Bogotá. ${ }^{28}$ El sociólogo Salvador Vidal-Ortíz, por su parte, está desarrollando un estudio sobre Colombia donde pretende identificar cuánto de la sexualidad tiene el desplazamiento forzado, así como reevaluar la definición institucional de "desplazamiento"

in Armed Conflict. En: Political Violence at a Glance, 2013. Disponible en: http://politicalviolenceataglance.org/2013/07/11/male-victimhoodin-armed-conflict/.

27

SIVAKUMARAN, Sandesh. Lost in translation: Un responses to sexual violence against men and boys in situations of armed conflict. En: International Review of the Red Cross, 2010.

28

PRADA, Nancy et al. “¡A mí me sacaron volada de ahí!”. 2012. 
pues, en su opinión, tiene un sesgo heterosexista que no ha permitido una aproximación distinta y más completa al tema. ${ }^{29}$ José Fernando Serrano realizó un trabajo sobre la invisibilidad de la diversidad de género y sexual en Colombia, así como de los procesos que se han adelantado en la construcción de paz a través de intervenciones directas de la ciudadanía para cambiar concepciones y actitudes desde la sociedad misma. ${ }^{30}$ En el marco del proceso de paz que adelanta el Gobierno colombiano con los guerrilleros de las FARC-EP, la organización Caribe Afirmativo presentó un breve informe sintetizando los problemas propios de la población LGBTI en el conflicto armado colombiano, y pidiendo que estos sean reconocidos y atendidos en el proceso que el Gobierno adelanta de reparación y memoria. ${ }^{31}$ A su vez, Colombia Diversa denun-

29 Vidal-Ortiz hace eco a una de las preocupaciones que motivan este artículo. En sus palabras: "La sexualidad, por ejemplo, incide en la definición de 'pureza' y 'decencia' que usan los grupos armados en la llamada 'limpieza social'. Las personas LGBT entran en un conjunto de categorías de personas estigmatizadas, junto a las trabajadoras sexuales y las personas que viven con $\mathrm{VIH}$ y Sida. Existen, incluso, usos nuevos de ese estigma y de la sexualidad en la guerra. Por ejemplo, se ha documentado cómo personas viviendo con VIH y Sida son movilizadas para usarlas como especie de 'armas biológicas' para que 'infecten' al enemigo. Son fenómenos nuevos, que demandan herramientas teóricas nuevas". CLAM. Sexualidad y Migración. Disponible en: http://www.clam.org.br/es/entrevistas/conteudo.asp?cod=8355

30 SERRANO, José Fernando. Paz, paso a paso. Agenciamiento e (in) visibilidad de la diversidad sexual y de género de la construcción de paz. Bogotá: CINEP, Universidad Javeriana, 2013.

31 Menciona el informe que "Ellas y ellos han sido víctimas de las formas de control moral y social impuestas por los actores violentos. Comunidades receptoras de personas desplazadas les ven como peligrosas o sospechosas, los grupos al margen de la ley han orientado hacia ellos su actuar violento y las restricciones al libre desarrollo de su personalidad, es una constante; reclamar sus derechos como víctimas les implica exponerse a la burla, la vergüenza y la discriminación con que aun se miran las sexualidades e identidades de género diversas en Colombia. Se trata de un balance difícil. Reconocerse como víctima, con el riesgo a ser estigmatizado por haber sido afectada por el conflicto, y reconocerse como gay, lesbiana, bisexual o transgenerista, con el riesgo de ser discriminado por la forma como aún se ve las identidades de género o las diversidades sexuales en sociedades permeadas cia las dificultades que se han presentado para la implementación de una política pública de reconocimiento y apoyo a las víctimas LGBTI. ${ }^{32}$

De la experiencia desarrollada por organizaciones como Colombia Diversa y de la literatura sobre violencia en el conflicto armado se pueden identificar obstáculos relacionados con (a) la situación de la víctima y su orientación sexual o identidad de género, (b) el tipo de violencia que sufre y (c) el contexto sociocultural en el cual ocurren los hechos.

\section{La situación de la víctima y su orienta- ción sexual o identidad de género}

Aún cuando las personas LGBTI son víctimas de los más diversos actos de violencia y discriminación existe un importante subregistro de estos hechos. Esto puede obedecer a factores institucionales, pero también tiene una importante relación con la identidad y con el poco apoyo que reciben las víctimas por parte de su familia o de su comunidad. En relación con la identidad de las personas LGBTI es importante señalar que muchas de ellas, víctimas de la violencia de actores armados, no han revelado su identidad

por el conflicto como la nuestra. Por ello, muchas de ellas prefieren invisibilizarse y terminan siendo presas de múltiples exclusiones y discriminaciones; niegan su orientación sexual, asumen prácticas de autocontrol en contra de su dignidad humana; se desplazan a donde no las conozcan o deciden invisibilizar su condición sexual, de género y como víctima. Así, unas formas de vulneración de derechos se suman a otras". CARIBE AFIRMATIVO. Caribe Afirmativo se une a la marcha de las víctimas del Caribe que se realizará en 23 de junio de 2013 en el Carmen de Bolívar e invita a la población LGBT a vincularse a este proceso ciudadano, 2013.

32 COLOMBIA DIVERSA. Situación de derechos humanos de la población Igbt en Colombia. Disponible en: http://www.oidhaco.org/ uploaded/content/article/902247002.pdf 
sexual en su entorno cercano o, si lo han hecho, ha sido a muy pocas personas. Esta situación es aún más grave en las zonas donde los actores armados aplican acciones de control social contra las comunidades, o en regiones más tradicionales o con mayor tradición religiosa. Por tanto, el miedo de las personas LGBTI a "salir del closet" es exacerbado por las amenazas, por el uso de la violencia armada o por otras condiciones socioculturales.

La población LGBTI que reside en zonas rurales tiene que enfrentarse también a otro problema: el sesgo urbano que afecta la identificación y protección de esta población. Luke Boso denuncia que las "narrativas culturales predominantes ubican geográficamente a las minorías sexuales en comunidades gays urbanas, dictan los contornos de cómo ser una persona gay moderna, e instan a las minorías sexuales para que salgan del closet y se asimilen en las culturas y comunidades gay". ${ }^{33}$ Lo que esto causa es invisibilizar a la población LGBTI rural en todos los debates sobre igualdad y sexualidad, borrando a las minorías más marginalizadas pues no se tiene en cuenta la creación de identidades en contextos donde factores como la familia, la religión y la comunidad de origen tienen una influencia preponderante. ${ }^{34}$ Esto termina en el desplazamien-

33 La traducción es nuestra. Texto en inglés: "Predominant cultural narratives geographically situate sexual minorities in urban gay communities, dictate the contours of how to be a modern gay person, and urge sexual minorities to come out and assimilate into gay communities and culture." BOSO, Luke A. Urban Bias, Rural Sexual Minorities, and the Courts. En: UCLA Law Review, 2013.

lbídem. to de la población LGBTı hacia las urbes o en aún más ocultamiento de esta en zonas rurales. ${ }^{35}$

Otra dificultad derivada de la situación de las víctimas se relaciona íntimamente con la anterior: la faceta social del ocultamiento. A causa de la orientación sexual y la identidad de género de las personas LGBTI, y por la discriminación y los prejuicios, muchas de ellas no cuentan con redes sociales fuertes que les den soporte y apoyo. Es común que sus familiares y amigos ignoren su condición o, si la conocen, sientan vergüenza y repudio. El ocultamiento de la orientación sexual e identidad de género, sumado a la falta de apoyo de su comunidad y a la ausencia de redes sociales, margina a las víctimas y reduce las probabilidades de que estas reclamen sus derechos y denuncien los abusos que se cometen en su contra. En consecuencia, la falta de instituciones adecuadas y la ausencia de redes sociales de apoyo son una barrera para el acceso a la justicia.

También en relación con la identidad, se destaca la imposibilidad o la dificultad de categorizar como LGBTI a personas que tienen prácticas sexuales no normativas o diferentes a las heterosexuales. En algunas regiones, y especialmente en las zonas rurales, las personas no heterosexuales no suelen reconocerse con una identidad tan definida, o lo hacen según variantes

35 Uno de los puntos que menciona Boso es el rol de los jueces en la difusión del sesgo urbano. Según el autor, los jueces en zonas rurales aceptan la idea de que la población Igbti no pertenece a esos espacios y que su hogar es la ciudad, por lo que terminan ejerciendo actos discriminatorios. Boso propone que es necesario reconocer la existencia de problemas propios en la población lgbti de las zonas rurales, de tal manera que la visibilización responda a la discriminación y entre a solucionar los problemas del sesgo. Ibídem. 
relacionadas con la actividad sexual. Por ejemplo, en el caso de los hombres, se cree que solo es homosexual quien tiene el papel "pasivo" en la relación sexual, es decir, quien es penetrado. La variación que los contextos culturales le imprimen a las identidades hace más difícil la caracterización de las víctimas, su visibilización y posterior reparación.

La población LGBTı no es homogénea en la definición de su identidad sexual ni en su reconocimiento público. Por ende, cualquier aproximación a estas víctimas debe ser sensible a las formas de identidades autodefinidas y a las dificultades de revelar la orientación sexual o identidad de género en escenarios que son inseguros no solo por el conflicto armado, sino también por las condiciones socioculturales de las comunidades.

\section{El tipo de violencia}

El segundo obstáculo tiene que ver con el tipo de violencia específica que se comete contra las personas LGBTI, en particular con la violencia por intolerancia, conocida erróneamente en el público como "limpieza social". ${ }^{36}$ En Colombia se ha investigado poco este tipo de violencia, aunque hay trabajos muy importantes de los inves-

36 Colombia Diversa reconoce que esta denominación de la violencia tiene un contenido en sí mismo discriminatorio y que tiende a justificar las acciones contra las comunidades marginadas. Lo conservamos porque es la forma como se conoce comúnmente y porque así lo han reportado diversos informes, denuncias y noticias al respecto. tigadores Juan Pablo Ordóñez, ${ }^{37}$ Carlos Rojas ${ }^{38}$ y Michael Taussig, ${ }^{39}$ entre otros. ${ }^{40}$

La violencia por intolerancia se dirige contra personas de comunidades históricamente discriminadas y socialmente vulnerables, especialmente contra habitantes de la calle, recicladores, trabajadoras y trabajadores sexuales, comunidad LGBTI, consumidores de droga, pequeños expendedores de droga y personas a quienes se acusa de cometer delitos como atracos y hurtos. Todos ellos comparten la situación de marginalidad y discriminación previa a la violencia. Por eso, diversas organizaciones de derechos humanos han documentado la violencia por intolerancia, sobre todo a partir de los años ochenta.

Este tipo de violencia es la expresión máxima de intolerancia social, y hunde sus raíces en la moralidad autoritaria que manejan algunos sectores de la comunidad. En algunos casos la comunidad comparte, aprueba o considera necesarios estos hechos de violencia por el rechazo visceral que las comunidades marginadas les inspiran. Por tanto, existe una complicidad táci-

37 No Human Being is Disposable: Social Cleansing, Human Rights, and Sexual Orientation in Colombia, Estados Unidos y Colombia, Colombian Human Rights Committee, International Gay and Lesbian Human Rights Comission y Proyecto Dignidad por los Derechos Humanos, 1995.

La violencia llamada ‘limpieza social’. Bogotá: CINEP, 1994

Law in Lawless Land: Diary of a Limpieza in Colombia. Chicago: The University of Chicago Press, 2005.

40 Véase, entre otros, STANNOW, Lovisa. Social Cleansing in Colombia. [Tesis Master of Arts]. Simon Fraser University, Burnaby, Canadá, 1996. GÓNGORA, Andrés y SUÁREZ, Carlos José. Por una Bogotá sin mugre: violencia, vida y muerte en la cloaca urbana. En: Revista Universitas Humanística, julio-diciembre del 2008, n. 66, p. 107-138. ROCHA, María Catalina. Estado de derecho, seguridad y marginalidad: representaciones en prensa sobre el fenómeno de la limpieza social en Colombia: 1988-1996. [Tesis de grado Maestría en Historia]. Pontificia Universidad Javeriana, 2009. 
ta entre comunidades y actores armados en la realización de estos hechos.

Otro aspecto relacionado con este tipo de violencia tiene que ver con las explicaciones que dan los actores armados frente a los homicidios de personas LGBTI. En muchos casos aseguran que las asesinan por razones distintas a las de su orientación sexual o identidad de género, y aducen la presunta comisión de delitos como el hurto o el abuso de niños y niñas. En una reciente versión libre del Proceso de Justicia y Paz, por ejemplo, el diario La Libertad, de Barranquilla, reportó la siguiente información:

En versión libre colectiva, el frente 'José Pablo Díaz' de las AUC reconoció el crimen de tres travestis en la calle 38. Según los desmovilizados, el triple crimen de los homosexuales se registró por una orden de 'El Loco Víctor', quien era el comandante del 'sector de la 40', y que el hecho fue cometido a eso de las 11:30 de la noche, porque tenían azotado el pedazo (sic) dándole burundanga a las víctimas para atracarlas y hurtarles sus pertenencias. Según uno de los versionados, en esos momentos había otros tres homosexuales. ${ }^{41}$

En el análisis de los patrones de victimización del frente "José Pablo Díaz", la Procuraduría remitió un informe pericial sobre los daños colectivos de estas comunidades. Al respecto dijo:

2.3.3.2 Los asesinatos que pretendían controlar la criminalidad: la limpieza social como práctica de ordenamiento social y moral.
Otra de las prácticas de victimización identificadas es la llamada "limpieza social" ejercida por el Frente José Pablo Díaz en contra de delincuentes comunes, consumidores de sustancias psicoactivas y Lesbianas, Gays, Bisexuales o Transgeneristas (LGBT). Este fenómeno de la limpieza social es complejo y problemático de analizar. Por lo general, los asesinatos de estas poblaciones que son vistas en ciertos contextos sociales y políticos como fuera de la estructura normal de la sociedad no se explican sólo a partir de la motivaciones construidas por los victimarios.

Como lo ha señalado Elsa Blair, existe un contexto social, cultural y político que produce estas víctimas y condiciona las características de su muerte. Por consiguiente, la limpieza social puede considerarse bajo esa doble perspectiva: a partir de las motivaciones de los victimarios y la del orden social y político que define las representaciones que se institucionalizan en la vida cotidiana sobre esas poblaciones. En este complejo entramado social y criminal que es la limpieza social, abordaremos dos prácticas de victimización que corresponden a la imposición de ciertos modelos ideológicos por parte del Frente José Pablo Díaz: en primer lugar los asesinatos que pretendían aparentemente controlar la criminalidad, entre ellos los asesinatos de personas con problemas de drogadicción, problemas mentales, mendigos, trabajadores ambulantes; y en segundo lugar, los asesinatos de la población LGBT". 42

41 "AUC reconoce muerte de ex alcalde de Santo Tomás y de tres travestis". La Libertad, junio 15 de 2010, p. 11-D.

PROCURADURÍA GENERAL DE LA NACIÓN. Informe pericial de daños colectivo a las comunidades victimizadas por el frente José Pablo Díaz del bloque norte de las Autodefensas Unidas de Colombia, 2011. 
Los victimarios no solo lesionan la memoria de las víctimas, ${ }^{43}$ sino que además crean una narración con la que tratan de justificar sus acciones y deslindarlas de los móviles discriminatorios. Al descartar los móviles homofóbicos contra la población LGBTI, los actores armados buscan que sus acciones ante la comunidad tengan una apariencia de justificación moral, social o política. Este tipo de justificaciones se asemejan en alto grado a las dadas en los casos de abuso policial u otras formas de violencia contra esta población.

Desentrañar los patrones de victimización de los procesos de violencia por intolerancia requiere un trabajo coordinado de las autoridades y varios movimientos sociales para tener mejor información y herramientas de análisis que permitan identificar dentro de los llamados "asesinatos selectivos" procesos más generales de victimización de comunidades.
43 La Comisión Colombiana de Juristas llamó la atención sobre esta situación en un reciente informe sobre el Proceso de Justicia y Paz: "[...] la manera como muchos paramilitares piden disculpas no resulta satisfactoria para las víctimas, pues algunos de los paramilitares postulados, como Ramón Isaza, alias ‘el Viejo', Camilo de Jesús Zuluaga, alias 'Napo' y José David Velandia Ramírez, alias 'Steven' del grupo paramilitar 'Autodefensas Campesinas del Magdalena Medio', tienen una especie de libreto para las versiones. Éste consiste en leer listas de nombres de personas asesinadas o desaparecidas por ellos, luego de lo cual mencionan rápidamente los supuestos motivos y justificaciones que tuvieron para asesinarlas o desaparecerlas (diciendo que eran auxiliadoras de la guerrilla, drogadictas, violadores, etcétera), y luego piden perdón a las víctimas. Esta forma de pedir disculpas no resulta reparadora, pues es mentirosa, justificatoria y poco genuina y no contribuye a hacer efectivo el derecho a la verdad". Véase COMISIÓN COLOMBIANA DE JURISTAS. Colombia: la metáfora del desmantelamiento de los grupos paramilitares. Segundo informe de balance sobre la aplicación de la Ley 975 del 2005. Bogotá, 2010, p. 136.

\section{El contexto sociocultural}

Por último, el tercer obstáculo se refiere a los contextos socioculturales en los que se produce la violencia contra la población LGBTI. En la mayoría de los casos, los hechos ocurren en comunidades que no aceptan la homosexualidad o que son abiertamente homofóbicas. Por esta razón, es importante indagar sobre la concepción que en las distintas zonas de violencia se tiene sobre la homosexualidad, y así identificar las causas, las consecuencias y la incidencia de estas concepciones en los hechos que afectan a la población LGBTI. En los estudios sobre la violencia contra las mujeres en el marco de conflictos armados, se ha acuñado el concepto “arreglos de género" ${ }^{44}$ para explicar la violen-

44 Este concepto ha sido ampliamente desarrollado por María Emma Wills, por ejemplo, en: Inclusión partidista y exclusión cultural en Colombia: pistas para comprender su relación. En: Análisis Político, mayo-agosto $2002, n^{\circ}$. 46. Historia, memorias, género: trayectoria de una iniciativa y aprendizajes, Fondo de Desarrollo de las Naciones Unidas para la Mujer (unifem): Justicia desigual. Género y derechos de las víctimas en Colombia, Bogotá: unifem Región Andina, junio de 2009, p. 41-85. Esta autora ha definido los "arreglos de género" como los "pactos legales o acuerdos informales mediante papeles en que se asignan ciertos atributos tanto para hombres como para mujeres dentro de un orden social determinado. A partir de las interacciones resultantes de estos pactos y acuerdos, se tienden a estructurar relaciones de poder específicas en las que, usualmente, se arroga más poder a lo masculino que a lo femenino. Esto termina por ser comprendido y reproducido por los actores sociales por cuanto 'natural' o 'biológico' aun cuando se ha construido culturalmente". CNNR/GRUPO DE MEMORIA HISTÓRICA. La masacre de Bahía Portete: mujeres Wayuu en la mira. En: Ediciones Semana y CNRR - Grupo de Memoria Histórica, 2010, p. 40. En los informes del mismo Grupo sobre las masacres de El Salado y de Trujillo se aplica este concepto, así como en el informe de las masacres de Chengue y del Tigre, de Alejandra Azuero para CODHES. Véase CNNR/GRUPO DE MEMORIA HISTÓRICA. Trujillo: una tragedia que no cesa. Bogotá: Planeta, 2008. CNNR/Grupo de Memoria Histórica. La masacre del Salado: esta guerra no es nuestra. Ediciones Semana y CNRR - Grupo de Memoria Histórica, 2009, p. 218, 227. AZUERO, Alejandra. Las sobrevivientes cuentan las experiencias de las mujeres en las masacres de Chengue y El Tigre. Consultoría para los Derechos Humanos y el Desplazamiento (codhes), Bogotá, 2009, p. 12. Otro análisis relevante es el de Ángela María Estrada et al. Regulación y control de la subjetividad y la vida privada en el contexto del conflicto armado colombiano. En: Revista de Estudios Sociales, junio 2003, n. 15, p. 133-149. 
cia, los factores que la potencian, e identificar los que en algunos casos la previenen. Estos "arreglos de género" podrían ser complementados con reflexiones sobre la sexualidad más allá de las concepciones binarias o heterosexistas, y de esta manera analizar arreglos de género y sexualidad que permitan prevenir la repetición de estos hechos.

En este mismo sentido, otro reto relacionado con los contextos son las variantes de la violencia contra la población LGBTI. La investigadora Elizabeth Wood señala la importancia de entender las variaciones de la violencia sexual, incluso teniendo en cuenta los casos en los que esta no ocurre, es decir, los "casos negativos". ${ }^{45}$ Esta variación, de acuerdo con el investigador William Payne, se hace extensiva a la comunidad LGBTI. Entender mejor las variaciones en relación con los grupos armados y los contextos en los que operan ayuda a caracterizar mejor el fenómeno; a formular medidas adecuadas de verdad, justicia y reparación; y a generar condiciones para su prevención. Muchos factores deben ser tenidos en cuenta para determinar la existencia y el grado de violencia contra la población LGBTI, como por ejemplo: (i) la presencia de una comunidad LGBTI organizada y reconocida por el resto de la comunidad; (ii) bajos niveles de homofobia y violencia previa; (iii) las concepciones morales de los comandantes e integrantes de los actores armados y las políticas internas de los grupos frente a la homosexualidad.

45 WOOD, Elisabeth. La violencia sexual en el marco de conflictos armados: hacia un entendimiento de su variación. En: Análisis Político, mayo-agosto 2009 , vol. $22, \mathrm{n}^{\circ} .66$.
También se deben tener en cuenta las variantes que obedecen a la dinámica y a la continuidad del conflicto armado y a sus actores. Es importante no perder de vista la creciente urbanización del conflicto, sobre todo por las consecuencias de la "desmovilización" de los grupos paramilitares, la creación de nuevas bandas criminales y otras variaciones del conflicto armado. Además, es relevante preguntarse por el impacto que en los actores armados han tenido la visibilidad y la movilización de la comunidad LGBTI. Como lo revela el trabajo de William Payne, informaciones periodísticas recientes ${ }^{46}$ y declaraciones políticas de grupos como la guerrilla de las FARC-EP, ${ }^{47}$ es posible que los avances legales y de derechos humanos de la población LGBTI tengan efectos en los actores armados, especialmente cuando estos tienen mayor contacto con las realidades urbanas, los medios de comunicación o las movilizaciones políticas. La continuidad del conflicto armado se da simultáneamente con una mayor visibilidad y empoderamiento de la población LGBTI, lo que también puede tener efectos, bien sea en el aumento o en la prevención de la violencia contra estas personas. Este nuevo estado de cosas es muy interesante, pues a partir de los avances legales y de derechos humanos se pueden articular propuestas que ayuden a crear herramientas con-

46 El programa Testigo Directo, de Caracol Televisión, presentó el caso de un reinsertado del Ejército de Liberación Nacional a quien durante su pertenencia en las filas se le permitió convivir con su compañero. "Un guerrillero salió del clóset en las filas del ELN". Disponible en: http://www.semana.com/noticias-conflicto-armado/guerrillero-saliodel-closet-filas-del-eln/135462.aspx [Última visita 14 de octubre de 2010].

47 Iván Márquez. Intervención en la Instalación de la Segunda Fase del Proceso de Paz, Disponible en: http://www.rebelion.org/noticia. php?id=157934 
tra la violencia, su prevención, y la garantía de la verdad, justicia y reparación de estas víctimas.

\section{$B$. Síntesis de la información sobre vio- lencia en el marco del conflicto armado contra la población LGBTI}

Esta sección sintetiza la información relacionada con los actos de violencia y de discriminación por parte de los actores armados contra las personas LGBTI. Antes, habría que subrayar que esta información es escasa si se compara con la documentación de otras situaciones de violación de derechos humanos de las personas LGBTI y frente a la información general sobre el conflicto armado, y que su documentación es notablemente informal. Sin embargo, en años recientes, gracias al trabajo de organizaciones con mayor arraigo local como Caribe Afirmativo y la Fundación Santamaría, entre otras, se ha mejorado la calidad de la información y la frecuencia de su denuncia. En otras palabras, la etapa de invisibilidad del fenómeno ha sido superada, pero aún son necesarios estudios que analicen los principales patrones de esta violencia.

A continuación nos referimos a la notable informalidad en la documentación del tema. Los hechos pueden obedecer al papel que los actores armados le atribuyen al "rumor" (por ejemplo en la "delación" y en otras formas de uso de la información pública y privada). ${ }^{48}$ Pero en el caso de las personas LGBTI en medio del conflicto armado, el rumor tiene dos efectos: los que se producen en su zona de residencia y en relación

48 ESTRADA, Fernando. La información y el rumor en zonas de conflicto. En: Análisis Político, mayo-agosto, 2007, n. 60, p. 44-59. con su orientación sexual o sus prácticas o comportamientos sexuales. Los comportamientos que se derivan de estos rumores están marcados por el prejuicio frente a las personas LGBTI: por ejemplo, acusar a un hombre gay de abusar sexualmente de niños y niñas. El rumor también puede ser el vehículo para reportar hechos de violencia. Esto ocurre principalmente por la ausencia de medios formales de comunicación. Finalmente, el rumor tiene un papel muy importante en la reconstrucción de la memoria sobre los hechos de violencia.

Así las cosas, el rumor puede ser generador de situaciones diversas: (i) rumores acerca de la sexualidad de una persona; (ii) rumores sobre su comportamiento sexual o social; (iii) rumores sobre la ocurrencia de hechos violentos contra las personas LGBT; (iv) el papel del rumor para la reconstrucción de la memoria. En los dos primeros casos, el rumor puede provocar el hecho violento -función constitutiva-; en los dos segundos, su función es difundir la información -función informativa-. El poder del rumor en la investigación de estos hechos no se debe subestimar, pero su análisis sigue siendo un reto para la documentación, especialmente en relación con su credibilidad y posibilidades de verificación a través de instrumentos de investigación social o judicial.

A manera de ejemplo, y como síntesis de la información relevante, en los siguientes apartes nos referimos a uno de los informes más importantes relacionados con la violencia contra la población LGBTı en el conflicto armado colombiano. 


\section{Cronología de la violencia en el contex- to del conflicto armado según el trabajo de Colombia Diversa}

En el Informe de derechos humanos de lesbianas, gais, bisexuales y transgeneristas en Colombia 2005, Colombia Diversa reportó varios hechos de violencia relacionados con el conflicto armado. El primero de ellos tenía que ver con la violencia por intolerancia, o "limpieza social". Sobre esta situación hay una tradición de denuncia -Oficina del Alto Comisionado de las Naciones Unidas para los Derechos Humanos y Comité de Derechos Humanos de Naciones Unidas- por lo que en ese entonces les fue muy útil el Informe de Colombia Diversa. Este se refería a una amenaza del 29 de septiembre del 2005 contra la comunidad LGBT del centro de la ciudad de Pereira. La había hecho un grupo de "limpieza social" autodenominado "Cazano". ${ }^{49}$ También se documentó la amenaza que había recibido una pareja de lesbianas por parte de presuntos integrantes de las Autodefensas Unidas de Colombia (Auc) en una vereda cercana a Ibagué, así como casos ocurridos en años anteriores: el homicidio de un hombre gay en el 2003, en el municipio de Caldas, Antioquia; el de una travesti en Campoalegre, Huila, el 15 de marzo del 2005; amenazas a un hombre gay y la desaparición forzada de su hermano en Barrancabermeja, Santander; el homicidio de tres

49 El panfleto decía, textualmente: "Señores Administradores: Este comunicado es con el fin de informarles que no queremos volver a ver maricas y prostitutas en la puerta de su negocio ni que les den servicio, si no cumplen esta orden en un tiempo límite de 3 días atengacen (sic) a las consecuencias que recaerán sobre ustedes. Grupo de Limpieza Urbano Cazano". travestis en Barranquilla, el 9 de septiembre del 2005.

El siguiente informe de derechos humanos de Colombia Diversa, correspondiente a los años 2006 y 2007, introducía un capítulo sobre la violencia por prejuicio y un anexo sobre la violencia vinculada al conflicto armado. El anexo presentaba los antecedentes de la violencia contra las personas LGBT en el marco del conflicto armado. En particular se destacaba el informe de Amnistía Internacional, Cuerpos marcados, crímenes silenciados, que había tenido una injerencia definitiva en la comprensión de la violencia basada en el género y la sexualidad. En relación con los hechos, y teniendo en cuenta el proceso de "desmovilización" y la dificultad de tener información de violaciones de derechos humanos contra la población LGBT por parte de los grupos guerrilleros, el anexo se concentró en los paramilitares.

El anexo del Informe de Colombia Diversa reporta un caso de amenaza colectiva en el centro de Pereira en el año 2006, relacionado con el que se había registrado en el informe anterior. Otro hecho particularmente grave fue el de la circulación de panfletos amenazantes contra la población LGBT en el municipio de Barrancabermeja, desde el 6 de julio del 2007, y el riesgo de desplazamiento que este generó. También se reportaron acciones de las llamadas "bandas emergentes de exparamilitares" contra la población LGBT en Medellín, Barrancabermeja, Bucaramanga y Pereira. Estos hechos fueron corroborados, incluso, por los defensores del pueblo de las regionales del Magdalena Medio, Risaralda y Arauca. 
En el período del que el presente informe se ocupa (2008 y 2009) se siguieron reportando hechos de violencia y de discriminación de los actores armados contra la población. Se destacan particularmente dos tipos de hechos: las amenazas colectivas de "limpieza social" contra personas LGBT y las acciones contra esta población cometidas presuntamente por paramilitares o por grupos formados por paramilitares desmovilizados.

Durante el año 2009, en todo el país, las amenazas y la intimidación contra comunidades marginales mediante panfletos que anunciaban actos violentos contra estas fue un fenómeno generalizado. ${ }^{50}$ Así lo informó la Oficina del Alto Comisionado de Naciones Unidas para los Derechos Humanos:

d) Entre febrero y marzo circularon panfletos con amenazas en por lo menos 24 de los 32 departamentos del país. Un formato estándar fue distribuido en poco más de dos semanas, en lugares tan distantes como Armenia, Barranquilla, Bogotá, Chocó, Cúcuta, Medellín y Valle del Cauca. El documento alentaba la mal llamada 'limpieza social' contra trabajadores sexuales, homosexuales, drogadictos y personas viviendo con $\mathrm{VIH}$, entre otros. El panfleto textualmente indicaba que '[quien] se encuentre [...] después de las 10:00 horas [en la calle] no responderemos', ordenaba '[...] esté más

50 Véase COALICIÓN CONTRA LA VINCULACIÓN DE NIÑOS, NIÑAS Y JÓVENES AL CONFLICTO ARMADO EN COLOMBIA (COALICO). Comunicado público. Bogotá, abril 7, 2009. COMISIÓN COLOMBIANA DE JURISTAS. La expresión de la violencia política y social a través de los panfletos paramilitares. Boletín $N^{\circ} 37$. Serie sobre los derechos de las víctimas y la aplicación de la Ley 975, 24 de julio del 2009. con la familia' y pedía 'perdón a la sociedad si caen inocentes' ${ }^{51}$

De hecho, los informes de alerta temprana de la Defensoría del Pueblo llamaban la atención sobre el riesgo que representaban los panfletos, así como mencionaban algunos hechos violentos que coincidían con su aparición. A pesar de que la Defensoría delegada para la producción de estos informes ${ }^{52}$ negó lo anterior, y sostuvo que en los informes de riesgo no existía información sobre la población LGBT, la organización Colombia Diversa tuvo acceso a algunos de ellos (los del departamento del Magdalena) y pudo verificar que, el 13 de mayo del 2009, al menos un hombre gay había sido asesinado y torturado en el casco urbano de Aracataca. ${ }^{53}$

En varias ciudades del país se han denunciado amenazas y homicidios perpetrados especialmente por grupos de paramilitares desmovilizados. En Cúcuta, el 5 de septiembre de 2009, se reportó una amenaza contra las travestis que ejercen prostitución en la zona céntrica de

51 Informe de la Alta Comisionada de las Naciones Unidas para los Derechos Humanos sobre la situación de los derechos humanos en Colombia, 4 de marzo del 2010.

52 La oficina encargada es la Defensoría Delegada para la Evaluación de Riesgos de la Población Civil como Consecuencia del Conflicto Armado. Es importante aclarar que el Sistema de Alertas Tempranas ha desarrollado herramientas sensibles al género. El Sistema incluye, de hecho, asuntos relacionados con la población LGBT y la homosexualidad, y en particular con la amenaza que pretende regular y controlar la vida cotidiana. Véase DEFENSORÍA DEL PUEBLO y UNIFEM. Porque el conflicto golpea... pero golpea distinto: herramientas para la apropiación de los indicadores de género del Sistema de Alertas Tempranas de la Defensoría del Pueblo para el monitoreo del conflicto armado. Bogotá: Defensoría del Pueblo, noviembre de 2007.

53 DEFENSORÍA DELEGADA PARA LA EVALUACIÓN DE RIESGOS DE LA POBLACIÓN CIVIL COMO CONSECUENCIA DEL CONFLICTO ARMADO, SISTEMA DE ALERTAS TEMPRANAS (SAT). Informe de riesgo $N^{\circ}$ 025-09A.I. Octubre 13 del 2009. 
la ciudad, y posteriormente el asesinato de tres de ellas. ${ }^{54}$ En Medellín, en el mes de agosto de ese mismo año, murió una pareja de lesbianas que vivían en una zona de la ciudad donde hacen presencia las llamadas bandas emergentes conformadas por desmovilizados. Así lo registraron los medios de comunicación: "En el barrio Santa Cruz, parte baja de la comuna nororiental de Medellín, fueron asesinadas a balazos las mujeres Janet Carmenza Amaya, de 35 años, y su pareja María Elena Murillo Caicedo, de 26, dos reconocidas líderes de la comunidad y de la población LGBT en Medellín". 55

En el informe "Impunidad sin fin" de la organización Colombia Diversa, que reporta los hechos violentos en el período 2010-2011, se revela un empeoramiento de las situaciones de violencia por prejuicio al menos en tres dimensiones. En primer lugar, para los años 2010 y 2011 se reporta la cifra más alta de homicidios contra población LGBTI desde que esa organización ha recogido datos (2006), con un total de 280 . En segundo lugar, de los 542 homicidios en total reportados entre el 2006 y el 2011, solo 20 han tenido sentencia condenatoria, es decir, menos del 5\%, y se evidencian investigaciones penales prejuiciadas y errores en el manejo de la evidencia. En tercer lugar, durante esos dos años se produjeron más amenazas a través de panfletos que en todos los períodos anteriores, como se muestra en la siguiente tabla tomada del informe de Colombia Diversa "Impunidad sin fin", 2013:

Tabla 1. Amenazas contra personas LGBT por año

\begin{tabular}{|c|c|c|}
\hline \multicolumn{2}{|c|}{ PANFLTOS AMENAZANTES POR DEPARTAMENTO } \\
\hline DEPARTAMENTO & PANFLETOS 2010 & PANFLETOS 2011 \\
\hline Atlántico & 2 & 4 \\
\hline Bolívar & 1 & 0 \\
\hline Cesar & 1 & 0 \\
\hline Córdoba & 1 & 1 \\
\hline Magdalena & 2 & 0 \\
\hline Norte de Santander & 1 & 2 \\
\hline Santander & 1 & 0 \\
\hline Sucre & 0 & 1 \\
\hline TOTAL PANFLETOS & 9 & 8 \\
\hline
\end{tabular}

Fuentes: Información de prensa, denuncias de organizaciones sociales, respuestas de personerías, defensorías y fiscalías a derechos de petición.

54 "Asesinadas tres personas de la comunidad LGBT en Cúcuta. Según indican líderes de la comunidad, se trata de muertes relacionadas con panfletos de los paramilitares". Caracol Radio. Audio de la noticia disponible en: http://www.caracol.com.co/programa. aspx?id=571008\&np rm=iDZ8W4eKn9bZuwEnRqa5xeeMsnC80YbQRNHVSUwX\%2BTelb UGx2YGquAaZH9nKOhST [Última visita 14 de octubre de 2010].

55 "Piden investigar el asesinato de dos integrantes de la comunidad LGBT en Medellín". Caracol Radio. 8 de agosto del 2010. Disponible en: http://www.caracol.com.co/nota.aspx?id=1351186 [Última visita 14 de octubre de 2010].
Un ejemplo de panfleto que circuló en la ciudad de Cúcuta en el año 2011 fue citado por la organización Colombia Diversa: 


\begin{abstract}
"HACEMOS ESTE COMUNICADO A TODOS LOS TRAVESTIS HIJUEPUTAS Y ASUS (SIC) CACORROS Y ALCAHUETAS DE ESOS HP

ESTE ES UN COMUNICADO PARA TODOS LOS HIJUPUTAS, TRAVESTIS, CACORROS, DROGADICTOS, CALLEJEROS, QUE SE ESTÁN INSTALANDO EN EL SCEPTOR(SIC) DE LA CALLE 7AV 3 Y A CELADORES QUE NO QUEREMOS VER MAS EN ESTA ZONA.

NADA DE PARCHES DE JOVENES NI LESVIANAS (SIC) FUERA DE LOS NEGOCIOS PORQUE VAMOS A PASAR EN ESTOS DIAS HACER (SIC) LIMPIEZA.

SE LES INFORMA A LOS NEGOCIOS QUE NO LES VENDA NADA A ESTAS PERSONAS NI LOS DEJE SENTAR ALAS (SIC) AFUERA (SIC) DE SU NEGOCIO PARA NO TENER QUE PERJUDICARLOS CUANDO FUCILEMOS (SIC) A ESTOS HIJUEPUTAS LA SEÑORA DE LOS PERROS SE TENDRÁ QUE IR POR SER COMPLICE DE LOS HIJUPUTAS Y CORRERA LOS MISMOS DESTINOS (SIC).

Y LOS NEGOCIOS DE CAFÉ JACK Y CROMOS. MAS ORDEN A LAS AFUERA (SIC) DE SU NEGOCIO"
\end{abstract}

Informaciones preliminares de Colombia Diversa reportan alrededor de 15 panfletos amenazantes en el 2012, casi el número total de los que se produjeron en los dos años anteriores, lo cual indica una permanencia e incluso un aumento en las amenazas contra la población LGBTI en el país.

En este informe también se analiza la condena contra el comandante paramilitar José Mogones Lugo, alias "Carlos Tijeras", por el homicidio de un hombre que, aunque no era gay, era percibido como tal por parte del grupo. Esto demuestra cómo los arreglos de género y sexualidad tienen una gran relevancia para la ocurrencia de un crimen, en este caso por presunta orientación sexual percibida por el actor armado. De hecho, en la sentencia judicial se establece la responsabilidad de "Carlos Tijeras" como máximo responsable de una "política de limpieza social" determinada por la empresa criminal. ${ }^{56}$

Si bien no todas las muertes y panfletos contra la población LGBTı pueden atribuirse al conflicto

56 COLOMBIA DIVERSA. Impunidad sin fin. Informe de derechos humanos de lesbianas, gay, bisexuales y personas trans en Colombia 2010 - 2011. 2013, p. 34-35. armado, también es cierto que no es posible decir lo contrario teniendo en cuenta el alto grado de impunidad que prevalece sobre estos hechos. Al respecto, es importante llamar la atención sobre el uso de los homicidios selectivos en el marco del conflicto armado, como lo documentó el Grupo de Memoria Histórica. Según el informe ¡Basta Ya!, “[I]os asesinatos selectivos constituyen la modalidad de violencia empleada por los actores armados que más muertos ha provocado en el desarrollo del conflicto". Luego continúa diciendo:

[l]os asesinatos selectivos son la expresión de una estrategia criminal que busca enmascarar las dimensiones de las acciones de violencia contra la población civil. Esto se debe a que su carácter de acción individual y su patrón de ataque dificultan la identificación de los perpetradores. La estrategia se complementa con un régimen de terror diseñado para silenciar a las víctimas y garantizar así la impunidad del crimen. ${ }^{57}$

En este sentido es posible que algunos de los homicidios contra población LGBTI, de los cua-

57 GRUPO DE MEMORIA HISTÓRICA. Op. cit. pp. 43-45. 
les no se ha identificado el móvil, o cuyo móvil sea el prejuicio, puedan tratarse de asesinatos selectivos realizados por actores armados. Recientemente se reportó que los paramilitares, en el marco de los procesos de Justicia y Paz, deberán responder por 624 casos de violencia sexual, entre los que se encuentran varios contra población LGBTI. ${ }^{58}$

En esta sección se abordaron los obstáculos para investigar la violencia contra las personas LGBTI en el marco del conflicto armado, y se presentó una síntesis de la información disponible sobre estas violaciones de derechos humanos. Como se muestra a lo largo de este relato, han sido las organizaciones y las víctimas quienes han dado visibilidad a la violencia como un presupuesto indispensable de la creación de un enfoque diferencial. El principal reto frente al enfoque diferencial es poder reconstruir los patrones de victimización de la población LGBTI. De esta manera se logrará consolidar un presupuesto fáctico más sólido para la atención, prevención y reparación de las víctimas de la violencia.

\section{EMERGENCIA DEL ENFOQUE DIFERENCIAL SOBRE ORIENTACIÓN SEXUAL EN LA LEY DE VÍCTIMAS}

La Ley de Víctimas incluye tres artículos que garantizan un enfoque diferencial para la población LGBTI: (i) el artículo 3 reconoce a las pare-

58 En la presentación de la noticia, El Tiempo destaca uno de los casos más crueles, relacionado con población Igbti: "En el 2001, paramilitares del Bloque Nutibara decidieron 'castigar' a un homosexual de una zona cerca de Medellín. Abusaron de él, lo empalaron y, finalmente, lo degollaron". Disponible en: http://www.eltiempo.com/justicia/ARTICULO-WEB-NEW_NOTA_INTERIOR-13068623.html jas del mismo sexo como víctimas en la misma mención a los familiares; (ii) el artículo 6 establece la cláusula de igualdad y no discriminación, reconociendo la orientación sexual como un criterio prohibido; y (iii) el artículo 13 establece el principio del enfoque diferencial, el cual "reconoce que hay poblaciones con características particulares en razón de su edad, género, orientación sexual y situación de discapacidad. Por tal razón, las medidas de ayuda humanitaria, atención, asistencia y reparación integral que se establecen en la presente ley, contarán con dicho enfoque". Estas tres normas son el fundamento para el enfoque diferencial de la población LGBTI.

Este enfoque diferencial surge como resultado de un proceso político que tiene al menos tres características. En primer lugar, el pronunciamiento judicial de la Corte Constitucional frente a la ampliación de las parejas del mismo sexo como víctimas en la sentencia C-029 de 2009, incluyendo las víctimas de la Ley de Justicia y Paz, desapariciones forzadas, proceso penal y daños civiles. Esta decisión judicial se produjo como resultado de un proceso de movilización legal para el reconocimiento de las parejas del mismo sexo. En segundo lugar, la Ley de Víctimas fue promovida por una coalición de actores políticos progresistas y movimientos sociales sensibles a los reclamos de la población LGBTI, que además habían compartido escenarios políticos con organizaciones de ese movimiento social. ${ }^{59}$ En tercer lugar, el trabajo de denuncia

59 Sobre el movimiento de víctimas, véase SANDOVAL, Nathalia. Movilizarse ante la Corte: trayectoria y efectos de tres episodios de movilización legal constitucional de feministas, indígenas y víctimas 
y documentación de las organizaciones LGBTI evidenció la violencia de la cual son víctimas estas comunidades.

Como resultado de este cambio legal, la Unidad para la Atención y Reparación Integral a las Víctimas (UARIV) ha iniciado acciones para implementar este enfoque diferencial. ${ }^{60}$ La primera acción fue crear el Grupo de Trabajo de Mujeres y Género a través de la Resolución 2043 de 2012, la cual sigue un concepto amplio de género que incluye "la identidad de género y las orientaciones erótico-afectivas diversas, lo que incluye a las personas LGBTI". La segunda acción fue la incorporación, en el formato único de declaración de víctima, la variable LGBTI, "desagregada por cada una de las identidades de género y orientaciones erótico-afectivas diversas". Esta variable permitió por primera vez la disponibilidad de datos oficiales sobre víctimas de la población LGBTı. Según la Unidad de Víctimas, entre el 10 de septiembre y el 31 de agosto de 2013 se han valorado 536 víctimas que se identifican a sí mismas como población LGBTI, de las cuales se incluyeron 374 en el registro. En total se han atendido 802 víctimas. En la tabla 2 compartimos las cifras de la Unidad, discriminadas por hecho victimizante contra la población LGBTI, y en la tabla 3 el número de personas atendidas. La tercera acción son los talleres y las capacitaciones que se han realizado con los funcionarios de la Unidad y aquellos encargados en atender

de crímenes de Estado en Colombia. [Tesis de Maestría en Ciencia Política]. Universidad de los Andes, 2012.

60 Todos los datos que a continuación se citan provienen de la respuesta de la Unidad de Víctimas a una petición realizada por la organización Caribe Afirmativo. Respuesta del 21 de junio de 2013. a las víctimas. Finalmente, la Unidad realizó, el 11 y 12 de septiembre, el primer "Encuentro nacional con personas LGBTI víctimas del conflicto armado". ${ }^{61}$ De dicho evento surgieron una serie de acuerdos enfocados en la comunicación y visibilización de la violencia contra la población LGBTI, en una mejor y más completa relación con las distintas instituciones encargadas de la documentación y reparación, y sobre el mejoramiento de las herramientas para la incidencia política de la población LGBTI. ${ }^{62}$

Tabla 2. Número de víctimas en el registro único de víctimas*

\begin{tabular}{|l|c|c|}
\hline \multicolumn{1}{|c|}{ Hecho victimizante } & $\begin{array}{c}\text { Total } \\
\text { registros } \\
\text { asociados }\end{array}$ & $\begin{array}{c}\text { Total registros } \\
\text { asociados con } \\
\text { reconocimiento }\end{array}$ \\
\hline $\begin{array}{l}\text { Acto terrorista / } \\
\text { atentados / combates } \\
\text { / enfrentamientos / } \\
\text { hostigamientos }\end{array}$ & 20 & 13 \\
\hline Amenaza & 109 & 54 \\
\hline $\begin{array}{l}\text { Delitos contra la libertad } \\
\text { y la integridad sexual en } \\
\text { desarrollo del conflicto } \\
\text { armado }\end{array}$ & 13 & 7 \\
\hline Desaparición forzada & 15 & 7 \\
\hline Desplazamiento forzado & 408 & 304 \\
\hline Homicidio & 106 & 56 \\
\hline
\end{tabular}

61 Según el acuerdo producto de dicho encuentro, en él participaron "ocho (8) mujeres lesbianas y bisexuales, dieciocho (18) hombres gay y bisexuales, y diez (10) mujeres trans, para un total de 36 personas, la gran mayoría integrantes de las mesas de participación de víctimas del nivel municipal y departamental, de 19 departamentos".

62 Este acuerdo estuvo acompañado de una estrategia de medios en donde se buscaba visibilizar el trabajo realizado. Se puede ver, por ejemplo, en http://www.elespectador.com/noticias/paz/y-si-dejamosel-estigma-articulo-446572, http://www.oim.org.co/noticias/96-fortalecimiento-institucional-para-la-atencion-a-victimas/2571-participacionde-victimas-Igbti-principal-logro-del-primer-encuentro-nacional.html y http://diarioadn.co/bogot\%C3\%A1/mi-ciudad/amenazas-a-miebrosde-comunidad-Igbti-1.76914. 


\begin{tabular}{|l|c|c|}
\hline \multicolumn{1}{|c|}{ Hecho victimizante } & $\begin{array}{c}\text { Total } \\
\text { registros } \\
\text { asociados }\end{array}$ & $\begin{array}{c}\text { Total registros } \\
\text { asociados con } \\
\text { reconocimiento }\end{array}$ \\
\hline $\begin{array}{l}\text { Minas antipersonal, } \\
\text { munición sin explotar } \\
\text { y artefacto explosivo } \\
\text { improvisado }\end{array}$ & 5 & 1 \\
\hline Secuestro & 9 & 3 \\
\hline Tortura & 7 & 1 \\
\hline $\begin{array}{l}\text { Vinculación de niños, } \\
\text { niñas y adolescentes a } \\
\text { actividades relacionadas } \\
\text { con grupos armados }\end{array}$ & 11 & 6 \\
\hline $\begin{array}{l}\text { Abandono o despojo } \\
\text { forzado de tierras }\end{array}$ & 7 & 6 \\
\hline Masivos & 715 & 461 \\
\hline TOTAL & 515 & \\
\hline
\end{tabular}

* Tomada de respuesta de Unidad de Víctimas sobre enfoque diferencial LGBTI.

Tabla 3. Número de personas atendidas*

\begin{tabular}{|l|l|}
\hline \multicolumn{1}{|c|}{ Tipo de atención prestada } & \multicolumn{1}{c|}{$\begin{array}{c}\text { Número de } \\
\text { víctimas LGBTI }\end{array}$} \\
\hline Atención humanitaria & 973 \\
\hline $\begin{array}{l}\text { Ayuda humanitaria para hechos } \\
\text { diferentes al desplazamiento }\end{array}$ & 1 \\
\hline $\begin{array}{l}\text { Extrema vulnerabilidad / Priorización } \\
\text { ayuda humanitaria }\end{array}$ & 1 \\
\hline Novedad solicitud & 4 \\
\hline Oferta institucional & 74 \\
\hline Peticiones, quejas y reclamos & 28 \\
\hline Registro & 94 \\
\hline Retorno connacional & 3 \\
\hline Retorno remisor & 2 \\
\hline Víctimas - Decreto 1290 & 59 \\
\hline Víctimas - Ley 1448 de 2012 & 15 \\
\hline Víctimas - Ley 418 de 1997 & 3 \\
\hline Total & 1257 \\
\hline
\end{tabular}

* Tomada de respuesta de Unidad de Víctimas sobre enfoque diferencial LGBTI.
Sin lugar a dudas, la creación de un enfoque diferencial para la población LGBTI ha sido un salto cualitativo en la forma como el Estado se aproxima a la violencia sufrida por este grupo. En otras palabras, el enfoque diferencial se ha convertido en el mecanismo discursivo e institucional que busca transformar la violencia, la invisibilidad y el odio de los actores armados en legitimación y dignidad para las víctimas.

Sin embargo, la implementación deficiente y los aspectos instrumentales de una política pública pueden afectar incluso los impactos simbólicos como el mencionado previamente. Por esta razón, los retos que plantea la violencia contra la población LGBTI y su investigación deben ser tomados en serio para que el enfoque diferencial sea un dispositivo institucional que garantice tanto efectos simbólicos como instrumentales. En la siguiente sección se plasman los retos que consideramos deben ser tenidos en cuenta para lograr la adecuada utilización del enfoque diferencial, así como la eficiente investigación sobre la violencia en contra de la población LGBTI.

\section{RETOS PARA EL ENFOQUE DIFERENCIAL Y LA INVESTIGACIÓN SOBRE LA VIOLENCIA CONTRA LA POBLACIÓN LGBTI}

Aquí se proponen tres retos relacionados con los obstáculos para la documentación que se expusieron en la sección primera, así como un reto adicional relacionado con la interacción institucional. 
El primer reto es la construcción y visibilización de la identidad de las víctimas LGBTI, la cual puede tener variaciones durante el proceso previo a la victimización, e incluso durante y después. La orientación sexual e identidad de género varían con el tiempo y las diversas situaciones personales. Por esta razón, las instituciones deben ser flexibles y fomentar espacios seguros para que las personas LGBTI puedan expresar su identidad de forma tranquila, incluso permitiendo no expresarla. Otra estrategia para fomentar espacios de confianza es crear programas de pares con otras personas LGBTI que puedan orientarlos adecuadamente tanto en la atención como en la reparación integral.

El segundo reto es el tipo de violencia que enfrentan las personas LGBTI, la continuidad de la violencia y su incremento en los últimos años. Cualquier proceso que pretenda dignificar a las víctimas debe asegurar en su conjunto la eliminación de las causas de la violencia, y garantizar que no se produzcan nuevas víctimas. Por esta razón, los procesos de paz y solución negociada al conflicto, así como el incremento de medidas de protección a estas comunidades, son urgentes para detener procesos de victimización, que como se describió previamente, van en aumento. Es además indispensable identificar los patrones de victimización, los actores, móviles, causas y consecuencias, y otros aspectos relacionados con la violencia que sufren las personas LGBTI. La mejor estrategia para superar este reto es desarrollar un estudio comprensivo que analice la información disponible y busque nueva información al respecto. En particular, es indispensable que las autoridades caractericen
Ios fenómenos de violencia por intolerancia, llamada equivocadamente "limpieza social".

El tercer reto es la intervención de contextos socioculturales discriminatorios, los cuales deben ser priorizados dentro de las políticas públicas. A partir de la caracterización de la violencia es posible identificar zonas y regiones donde la discriminación y la homofobia son más prevalentes, y donde estas tuvieron impacto en la producción y justificación de la violencia. La mejor forma de abordar este reto es priorizando estas zonas como áreas de intervención de las políticas públicas locales y nacionales sobre derechos LGBTı y campañas de convivencia con la diferencia por razón de la orientación sexual e identidad de género. La mayoría de políticas públicas de derechos LGBTI se concentran en grandes centros urbanos y descuidan lugares rurales u otros centros urbanos donde el conflicto armado ha sido más fuerte.

Finalmente, el cuarto reto se refiere a la acción del Estado en su conjunto. El enfoque diferencial no puede ser un mecanismo únicamente de la Unidad de Víctimas, sino que debe ser parte de una política integral del Estado que incluya instituciones como la Fiscalía General de la Nación, la Procuraduría General de la Nación, las fuerzas militares, los jueces, el Congreso y, en general, todas las ramas del poder público. El problema de aislar el enfoque diferencial como una función de una sola agencia crea problemas tanto para la atención como para la reparación integral. Así, por ejemplo, una indebida investigación judicial hace inoperantes las medidas de reparación integral, o una oposición 
abierta a los derechos LGBTI por parte de alguna institución termina contribuyendo a fenómenos de violencia institucional o por parte de actores armados.

\section{CONCLUSIÓN}

El enfoque diferencial para la población LGBTI surge de un proceso político complejo que tuvo como antecedente la emergencia de un discurso y una práctica política de denuncia y documentación de la violencia contra las personas LGBTI en Colombia. Durante este proceso de documentación, las organizaciones LGBTI han evidenciado obstáculos importantes para identificar y sistematizar estos hechos de violencia, bien sea por las condiciones de las personas afectadas, el tipo de violencia que sufren, o los contextos socioculturales en los cuales se producen los actos violentos. A pesar de estos obstáculos, existe un importante punto de partida para continuar analizando la violencia contra la población LGBTI y buscar soluciones que permitan prevenirla, así como la atención y reparación de las víctimas. Sin lugar a dudas, el enfoque diferencial es una herramienta de política pública que puede transformar la violencia y el silencio en el cual ocurre, y buscar caminos para dignificar a las víctimas. Para ello se requieren acciones que fortalezcan la identidad de las víctimas, que aborden integralmente la violencia por intolerancia y la creación de políticas públicas para garantizar la igualdad y la diversidad por orientación sexual e identidad de género. $\mathrm{E}$ enfoque diferencial para la población LGBTI en la Ley de Víctimas tiene potencialidades transfor- madoras, pero esto solo ocurrirá si el Estado en su conjunto actúa a favor de la igualdad y el reconocimiento de la diversidad sexual. En este punto, la directriz 9 del Alto Comisionado de las Naciones Unidas para los Refugiados da recomendaciones que son muy útiles para definir el camino adecuado a recorrer. Debe comprenderse que: (1) la falta de información es usual en estos casos, y que eso no es causal para desestimar a los solicitantes de ayuda o para pensar que en efecto no hay víctimas; (2) el ocultamiento de la orientación es común, particularmente cuando se viene de contextos de opresión social y criminalización paraestatal (común en el caso colombiano), por lo que deben identificarse los factores determinantes según el contexto (como la religión y el contexto sociocultural, por ejemplo), y usarlos en la construcción de la narrativa de la violencia, teniendo en cuenta que muchas veces las personas están cohibidas por la vergüenza y el tabú; (3) las categorías LGBTı son flexibles y en ocasiones las personas no se identifican con ellas, por lo que la existencia de estas no puede convertirse en un obstáculo para la reparación de los casos de difícil categorización; (4) la sensibilidad es clave en todo el proceso de identificación y reparación para no causar revictimización; (5) se requiere un esfuerzo institucional integral para comprender la problemática y atenderla de manera adecuada; y (6) debe hacerse un esfuerzo consciente por romper el sesgo urbano y atender a las necesidades de las poblaciones rurales donde el conflicto armado tiende a tener efectos más fuertes.

Si no se logra la construcción de un esfuerzo con las características que expusimos, este 
enfoque diferencial corre el riesgo de ser un discurso, que incluso cuando habla, oculta las causas estructurales de la discriminación que no residen únicamente en la mente de un actor armado, sino podrían estar arraigadas en nuestras instituciones o incluso en nuestras mentes y corazones.

\section{Bibliografía}

ALBARRACÍN, Mauricio. Desplazamiento forzado contra las personas LGBT: reflexiones para la investigación e implementación de políticas públicas. En: Colombia Diversa. Todos los deberes, pocos los derechos: situación de las personas lesbianas, gay, bisexuales y transgeneristas 2008-2009. Bogotá, 2011.

ALTO COMISIONADO DE LAS NACIONES UNIDAS PARA LOS REFUGIADOS -ACNUR. Directrices sobre protección internacional $n^{\circ}$. 9. Disponible en: http://www.refworld. org/cgi-bin/texis/vtx/rwmain/opendocpdf. pdf? reldoc $=y \& d o c i d=518113 d 54$

AZUERO, Alejandra. Las sobrevivientes cuentan las experiencias de las mujeres en las masacres de Chengue y El Tigre. Consultoría para los Derechos Humanos y el Desplazamiento (CODHES). Bogotá, 2009, p. 12.

BERG, Laurie y MILLBANK, Jenni. Constructing the Personal Narratives of Lesbian, Gay and Bisexual Asylum Claimants. En: Journal of Refugee Studies, 2009, vol. 22, nº. 2.

BOSO, Luke A. Urban Bias, Rural Sexual Minorities, and the Courts. En: ucla Law Review. 2013.
BOURDIEU, Pierre. La dominación masculina. Barcelona: Anagrama, 1998.

CARACOL RADIO. Audio de la noticia disponible en: http://www.caracol.com.co/programa.aspx ?id=571008\&nprm=iDZ8W4eKn9bZuwEnRqa5 xeeMsnC80YbQRNHVSUwX\%2BTelbUGx2YGqu AaZH9nKOhST.

CARACOL RADIO. Piden investigar el asesinato de dos integrantes de la comunidad LGBT en Medellín. 8 de agosto del 2010. Disponible en: http:// www.caracol.com.co/nota.aspx?id=1351186.

CARIBE AFIRMATIVO. Caribe Afirmativo se une a la marcha de las víctimas del Caribe que se realizará en 23 de junio de 2013 en el Carmen de Bolívar e invita a la población LGBT a vincularse a este proceso ciudadano. 2013.

CARPENTER, Charli. Recognizing Gender-Based Violence Against Civilian Men and Boys in Conflict Situations. En: Security Dialogue, March 2006, vol. 37, pp. 183-103. Disponible en http://sdi. sagepub.com/content/37/1/83.short

CENTRO LATINOAMERICANO DE SEXUALIDAD Y DERECHOS HUMANOS -CLAM+10. Sexualidad y migración. Disponible en: http://www.clam.org. br/es/entrevistas/conteudo.asp?cod=8355

CNNR/GRUPO DE MEMORIA HISTÓRICA. La masacre de Bahía Portete: mujeres Wayuu en la mira. En: Ediciones Semana y CNRR - Grupo de Memoria Histórica. 2010.

CNNR/GRUPO DE MEMORIA HISTÓRICA. TrujiIlo: una tragedia que no cesa. Bogotá: Planeta, 2008. 
CNNR/GRUPO DE MEMORIA HISTÓRICA. La masacre del Salado: esta guerra no es nuestra.

COALICIÓN CONTRA LA VINCULACIÓN DE NIÑOS, NIÑAS Y JÓVENES AL CONFLICTO ARMADO EN COLOMBIA (COAlico). Comunicado público. Bogotá, abril 7, 2009.

COLOMBIA DIVERSA. Impunidad sin fin. Informe de derechos humanos de lesbianas, gay, bisexuales y personas trans en Colombia 2010 - 2011, 2013.

COLOMBIA DIVERSA. Situación de derechos humanos de la población LGBT en Colombia. Disponible en: http://www.oidhaco.org/uploaded/ content/article/902247002.pdf

COLOMBIA. Ley 1448 de 2011.

ESPAÑA. Ley 52 de 2007. Ley de Memoria Histórica de España.

COMISIÓN COLOMBIANA DE JURISTAS. COlombia: la metáfora del desmantelamiento de los grupos paramilitares. Segundo informe de balance sobre la aplicación de la Ley 975 del 2005. Bogotá, 2010.

COMISIÓN COLOMBIANA DE JURISTAS. La expresión de la violencia política y social a través de los panfletos paramilitares. Boletín $n^{\circ}$. 37. Serie sobre los derechos de las víctimas y la aplicación de la Ley 975, 24 de julio del 2009.

COMISIÓN DE LA VERDAD Y RECONCILIACIÓN. Informe final. Tomo II. Perú, 2003.
DAUVERGNE, Catherine y MILLBANK, Jenni. Burdened by Proof: How the Australian Refugee Review Tribunal Has Failed Lesbian and Gay Asylum Seekers. En: Federal Law Review, 2003, 31, p. 299-342.

DEFENSORÍA DEL PUEBLO y UNIFEM. Porque el conflicto golpea... pero golpea distinto: herramientas para la apropiación de los indicadores de género del Sistema de Alertas Tempranas de la Defensoría del Pueblo para el monitoreo del conflicto armado. Bogotá: Defensoría del Pueblo, noviembre de 2007.

DEFENSORÍA DELEGADA PARA LA EVALUACIÓN DE RIESGOS DE LA POBLACIÓN CIVIL COMO CONSECUENCIA DEL CONFLICTO ARMADO, SISTEMA DE ALERTAS TEMPRANAS (SAT). Informe de riesgo $n^{\circ}$. 025-09A.I. Octubre 13 del 2009.

DIARIO ADN. Miedo en víctimas del conflicto por hacer parte de la comunidad LGBTI. 16 de septiembre de 2013. Disponible en: http://diarioadn.co/bogot\%C3\%A1/mi-ciudad/amenazas-amiebros-de-comunidad-lgbti-1.76914.

ECK, Kristine y HÄNNI, Olga. Male Victimhood in Armed Conflict. En: Political Violence at a Glance. 2013. Disponible en http://politicalviolenceataglance.org/2013/07/11/male-victimhoodin-armed-conflict/.

EL ESPECTADOR. Y si dejamos el estigma... 15 de septiembre de 2013. Disponible en: http:// www.elespectador.com/noticias/paz/y-si-dejamos-el-estigma-articulo-446572. 
EL TIEMPO. Cúpula de Auc, a responder por 624 crímenes sexuales. 17 de septiembre del 2013. Disponible en: http://www.eltiempo. com/justicia/ARTICULO-WEB-NEW_NOTA_INTERIOR-13068623.html

EPPS, Bradley S. Proper Conduct: Reinaldo Arenas, Fidel Castro, and the Politics of Homosexuality. En: Journal Of the History of Sexuality, 1995, n. 66.

ERIBON, Didier. Reflexiones sobre la cuestión gay. Barcelona: Anagrama, 2001.

ESGUERRA, Camila. A propósito de la vergüenza en prácticas artísticas enfoques contemporáneos. Bogotá: Universidad Nacional - IDCT, 2003.

ESTRADA, Ángela María et al. Regulación y control de la subjetividad y la vida privada en el contexto del conflicto armado colombiano. En: Revista de Estudios Sociales, junio 2003, nº 15.

ESTRADA, Fernando. La información y el rumor en zonas de conflicto. En: Análisis Político, mayo-agosto $2007, n^{\circ} .66$.

FAIRBURN, Bill. Gay Rights Are Human Rights: Gay Asylum Seekers in Canada. En: E. Brad, K. Valens and B. J. González (editors). Passing Lines: Sexuality and Immigration. Cambridge, MA: David Rockefeller Center for Latin American Studies, Harvard University, 2005, pp. 237-252.

GILES, Geoffrey J. Legislating Homophobia in the Third Reich: The Radicalization of Prosecu- tion Against Homosexuality by the Legal Profession. En: German History, 2005, vol. 3, nº 23.

GÓNGORA, Andrés y SUÁREZ, Carlos José. Por una Bogotá sin mugre: violencia, vida y muerte en la cloaca urbana. En: Universitas Humanística, julio-diciembre 2008, n. 66, p. 107-138.

GRUPO DE MEMORIA HISTÓRICA. ¡Basta ya! Colombia: memorias de guerra y dignidad. Bogotá: Imprenta Nacional, 2013.

HANNA, Fadi. Punishing Masculinity in Gay Asylum Claims. En: The Yale Law Journal, January 2005, n. 114.

KENDALL, Christopher N. Lesbian and Gay Refugees in Australia: Now that "Acting Discreetly" Is No longer an Option, Will Equality Be Forthcoming? En: International Journal of Refugee Law, 2005, vol. $15, n^{\circ} .4$.

LA LIBERTAD. AUC reconoce muerte de ex alcalde de Santo Tomás y de tres travestis. Barranquila, 15 de junio de 2010, p. 11-D.

LEMAITRE RIPOLL, Julieta. El amor en tiempos de cólera: derechos LGBT en Colombia. En: SUR Revista Internacional de Derechos Humanos, dic. 2009 , vol. 6 , no. 11.

LEWIS, Dustin. Unrecognized Victims: Sexual Violence Against Men in Conflict Settings Under International Law. En: Wisconsin International Law Journal, 2009.

MÁRQUEZ, Iván. Intervención en la instalación de la segunda fase del Proceso de Paz. Dis- 
ponible en: http://www.rebelion.org/noticia. php?id=157934

MCGHEE, Derek. Persecution and Social Group Status: Homosexual Refugees in the 1990s. En: 14 J. Refugee Studies 20, 2001.

MILLBANK, Jenni. Constructing the Personal Narratives of Lesbian, Gay, and Bisexual Asylum Claimants. En: Journal of Refugee Studies, 2009.

OFICINA DEL ALTO COMISIONADO PARA LOS DERECHOS HUMANOS. Informe de la Alta Comisionada de las Naciones Unidas para los Derechos Humanos sobre la situación de los derechos humanos en Colombia, 2009. Washington, 4 de marzo del 2010.

OOSTERHOFF, Pauline; ZWANIKKEN, Prisca y KETTING, Evert. Sexual Torture of Men in Croatia and Other Conflict Situations: An Open Secret. En: Reproductive Health matters. May 2004, vol. 12, p. 68-77. Disponible en http://www.rhmelsevier.com/article/S0968-8080(04)231159/abstract.

ORGANIZACIÓN INTERNACIONAL PARA LAS MIGRACIONES. Participación de víctimas LGBTI, principal logro del primer encuentro nacional. 17 de septiembre de 2013. Disponible en: http:// www.oim.org.co/noticias/96-fortalecimientoinstitucional-para-la-atencion-a-victimas/2571participacion-de-victimas-Igbti-principal-logrodel-primer-encuentro-nacional.html.

PRADA, Nancy, et al. ¡A mí me sacaron volada de ahí!. 2012.
PROCURADURÍA GENERAL DE LA NACIÓN. Informe pericial de daños colectivos a las comunidades victimizadas por el frente José Pablo Díaz del bloque norte de las Autodefensas Unidas de Colombia, 2011.

PROUST, Marcel. En busca del tiempo perdido. 1913.

REHAAG, Sean. Patrolling the Borders of Sexual Orientation: Bisexual Refugee Claims in Canada. En: McGill Law Journal, 2008, nº 53.

ROCHA, María Catalina. Estado de derecho, seguridad y marginalidad: representaciones en prensa sobre el fenómeno de la limpieza social en Colombia: 1988-1996. [Tesis de grado Maestría en Historia]. Pontificia Universidad Javeriana, 2009.

ROJAS, Carlos. La violencia llamada 'limpieza social'. Bogotá: Cinep, 1994.

SANDOVAL, Nathalia. Movilizarse ante la Corte: trayectoria y efectos de tres episodios de movilización legal constitucional de feministas, indígenas y víctimas de crímenes de Estado en Colombia. [Tesis de Maestría en Ciencia Política]. Univesidad de los Andes, 2012.

SEMANA. Un guerrillero salió del closet en las filas del ELN. 24 de febrero de 2010. Disponible en: http://www.semana.com/noticias-conflictoarmado/guerrillero-salio-del-closet-filas-deleln/135462.aspx.

SERRANO, José Fernando. Paz, paso a paso. Agenciamiento e (in)visibilidad de la diversidad 
sexual y de género de la construcción de paz. Bogotá: Cinep, Universidad Javeriana, 2013.

SIVAKUMARAN, Sandesh. Lost in translation: Un responses to sexual violence against men and boys in situations of armed conflict. En: International Review of the Red Cross, 2010.

SIVAKUMARAN, Sandesh. Male/Male Rape and the "Taint" of Homosexuality. En: Human Rights Quarterly, 2005.

SOUTH AFRICAN MEDICAL RESEARCH COUNCIL. Briefing Paper. Care and Support of Male Survivors of Conflict-Related Sexual Violence. Sexual Violence Research Initiative. 2011. Disponible en http://www.svri.org/CareSupportofMaleSurviv.pdf.

STANNOW, Lovisa. Social Cleansing in Colombia. [Tesis Master of Arts]. Simon Fraser University, Burnaby, Canadá, 1996.

SWINK, Arwen. Queer Refuge: A Review of the Role of Country Condition Analysis in Asylum Adjudications for Members of Sexual Minorities. En: 29 Hastings Int'l \& Comp. L. Rev., 2006.

TAUSSIG, Michael. Law in Lawless Land: Diary of a Limpieza in Colombia. Chicago: The University of Chicago Press, 2005.

U. S. CITIZENSHIP AND INMIGRATION SERVICES. Guidance for adjudicating Lesbian, Gay, Bisexual, Transgender, and Intersex (LGBTI) refugee and asylum claims, 2011.
UNITED NATIONS HIGH COMMISSIONER FOR REFUGEES -UNHCR. UNHCR Guidance note on refugee claims relating to sexual orientation and gender identity. Geneva, November 2008.

WILLS, María Emma. Historia, memorias, género: trayectoria de una iniciativa y aprendizajes. En: Justicia desigual. Género y derechos de las víctimas en Colombia. Bogotá: unifem-Región Andina, junio de 2009.

WILLS, María Emma. Inclusión partidista y exclusión cultural en Colombia: pistas para comprender su relación. En: Análisis Político, mayoagosto 2002, $n^{\circ} .46$.

WOOD, Elisabeth. La violencia sexual en el marco de conflictos armados: hacia un entendimiento de su variación. En: Análisis Político, mayo-agosto, 2009, vol. 22, nº 66.

ZARKOV, Dubrabvka. The Body of The Other Man. Sexual violence and the Construction of Masculinity, Sexuality and Ethnicity in Croatian Media. En: C. MOSER y F. CLARK. Victims, Perpretators, or actors? Gender, Armed Conflict and Political Violence. Capítulo 5. Zed Books. Disponible en: http://www.umass.edu/wost/syllabi/spring06/ zarkov.pdf

ZAWATI, Hilmi. Impunity or immunity: wartime male rape and sexual torture as a crime against humanity. En: Journal on Rehabilitation of Torture Victims and Prevention of Torture. P. 27-47. Disponible en: http://www.irct.org/Files/Filer/ TortureJournal/17_1_2007/impunity_or_immunity.pdf 